\title{
Trématodes Digenea adultes \\ chez des Batraciens, Reptiles, Oiseaux
}

\author{
Par Robert-Ph. DOLLFUS
}

La collection de Trématodes digénétiques récoltés en Iran par mes amis les docteurs Golvan et Rioux comprend 11 espèces. Ce sont toutes des espèces paléarctiques, antérieurement décrites et dont la présence était connue en U.R.S.S., particulièrement en Asie russe. Au point de vue biogéographique, il est intéressant de savoir qu'elles existent aussi dans la faune iranienne, encore actuellement peu connue en ce qui concerne les Trématodes.

Au point de vue morphologique, l'étude de ce matériel a permis une connaissance plus étendue de plusieurs espèces.

\section{Famille CYCLOCOELIDAE W. Kossack 1911 Sous-Famille haEmatotrephinaE R. - Ph. Dollfus 1948 HAEMATOTREPHUS (UVITELLINA) VANELLI (Rudolphi 1819) R. - Ph. Dollfus 1948}

1819. « Monostoma Vanelli » C. A. Rudolphi, p. 87, 350.

1933. «Uvitellina tageri n. sp. »: S. Yamaguti, p. 48-50, fig. 21.

1948. « Haematotrephus (Uvitellina) vanelli (Rudolphi 1819) »: R.-Ph. Dollfus, p. 146, 147, 177, fig. 3 .

1959. « Uvitellina tageri Yamaguti $1933=$ Cyclocoelum (Haematotrephus) lanceolatum (Wedl 1858) »: G. Dubois, p. 96.

1962. « Uvitellina keri Yamaguti (syn. U. tageri Yamaguti, 1933) »: I. Bychovskaïa-Pavlovskaïa, p. 109, 302.

\section{Matériel de la collection :}

Un spécimen mesurant $21 \times 4,5 \mathrm{~mm}$ de la cavité thoracique de Vanellus vanellus (L. 1758) (Charadrii), à Akinlou, 5-10-1959. 
Je conserve Uvitellina G. Witenberg 1923 comme sous-genre, mais il a été supprimé et placé en synonymie d'Haematotrephus M. Stossich 1902 par G. Dubois (1959, page 76).

G. Dubois (1959, p. 96) n'accepte pas vanelli (Rudolphi) comme nom d'espèce, parce que l'on a trouvé, accidentellement, chez le vanneau, Cyclocalum (Cyclocalum) obscurum (J. Leidy 1887) et Cyclocoelum (Haematotrephus) tringae M. Stossich 1902 ; en outre, G. Dubois (1959, p. 110) place Monostoma Vanelli Rud. dans ses « species delineatae », le Muséum de Vienne ne possédant plus d'exemplaire de l'espèce de Rudolphi.

J'estime qu'il est toujours préférable de reprendre un nom ancien en lui donnant une acception précise plutôt que de l'abandonner définitivement, et je considère Uvitellina tageri Yamaguti comme néotype de l'espèce de Rudolphi.

\section{Famille ECHINOSTOMATIDAE E. Dietz 1909 Sous-Famille ECHINOCHASMINAE T. Odhner 1910 MESORCHIS PSEUDOECHINATUS (P. Olsson 1876), var.}

(Fig. 1-3)

\section{Matériel de la collection :}

Environ 50 individus, de l'intestin grêle d'un Podiceps cristatus (L. 1758) de Bendar-Pahlevi, au bord de la Mer Caspienne, 14-12-1959.

\section{Description :}

Corps très allongé, étroit, grêle, mesurant, par exemple, $3,7 \times 0,5,4,2 \times 0,46$, $5,2 \times 0,6 \mathrm{~mm}$. Cuticule ornée d'écailles à bord libre arrondi en vue de face, mais à aspect d'épines en vue de profil ; se raréfiant et s'espaçant en direction postérieure pour disparaître complètement en arrière des testicules.

Ventouse orale $0,136 \times 0,160$. Les épines céphaliques sont au nombre de 22 , elles mesurent $(\mu)$, en comptant comme première la plus antérieure de chaque côté : $1^{\text {re }}: 84 \times 20 ; 2^{\circ}: 74 \times 20 ; 6^{\circ}: 80 \times 20 ; 7^{\circ}: 88 \times 23 ; 8^{\circ}: 93 \times 20 ; 10^{\circ}: 80 \times$ $15,6: 11^{\circ}: 70,2 \times 15,6$. Il y a interruption au milieu du bord antérieur. Prépharynx, 0,08 ; pharynx, $0,148 \times 0,136$; œsophage, 0,378 ; ventouse ventrale, diamètre 0,394 ; ovaire globuleux, non lobé, diamètre 0,171 ; testicules ovales, non lobés, l'antérieur $0,39 \times 0,314$, le postérieur $0,43 \times 0,314 \mathrm{~mm}$. La poche du cirre est ovale, $0,234 \times$ $0,156 \mathrm{~mm}$, en partie dorsale à la ventouse ventrale, en partie en avant ; le pore génital est submédian, à environ $0,107-0,130$ en avant du bord antérieur de la ventouse ventrale ; il est entouré de fines épines de 8 à $12 \mu$, paraissant disposées sur plusieurs rangs. Ces épines sont probablement caduques, n'étant pas observables chez tous les individus. Les vitellogènes débutent entre le niveau de la mi-longueur ou du quart postérieur du 


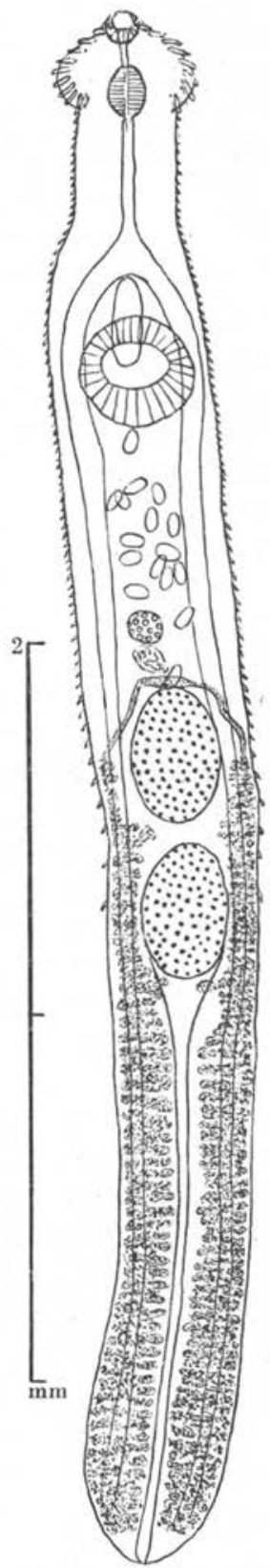

FIG. 1. - Mesorchis pseudoechinatus ( $\mathrm{P}$. Olsson 1876), var. Intestin. Podiceps cristatus (L.). Bendar-Pahlevi (Iran). Y. Golvan 14.12.1959.

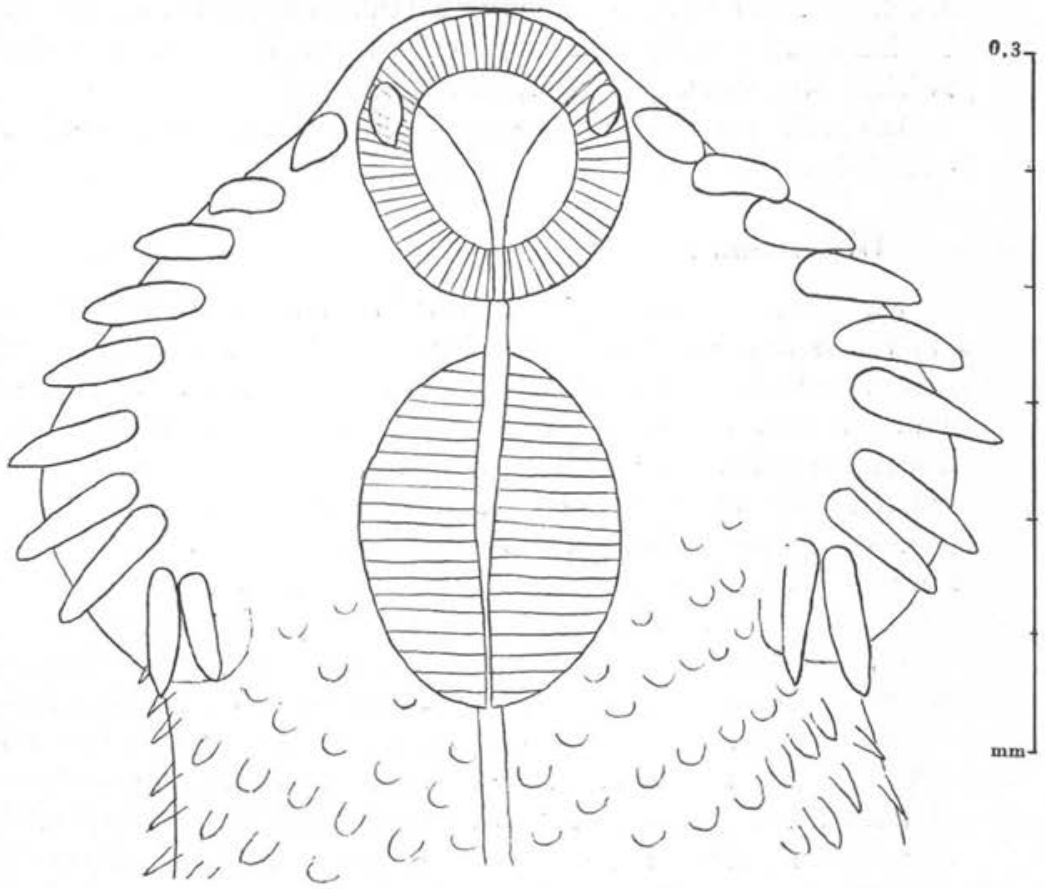

Fı. 2. - Epines céphaliques d'un individu de même provenance que celui de la fig. 1

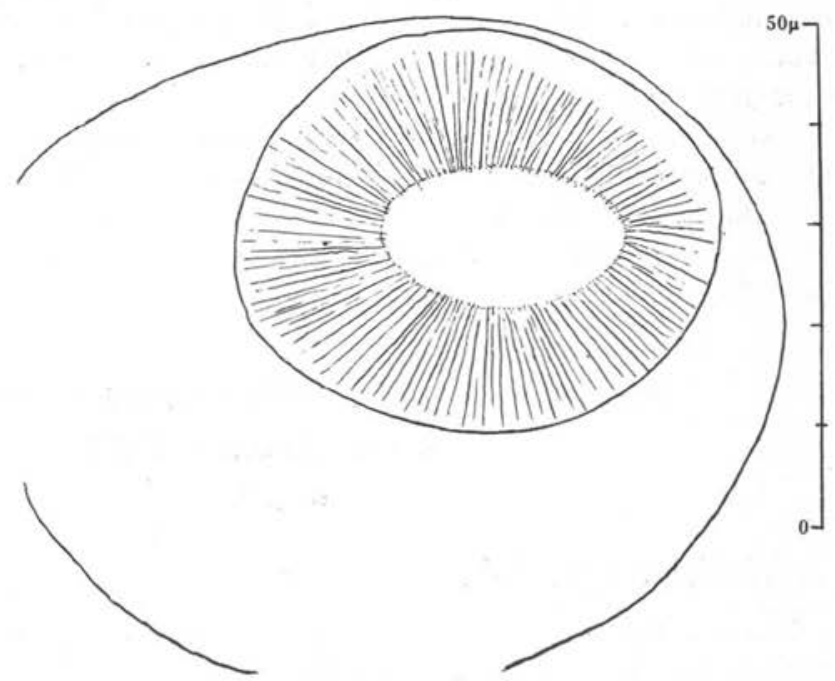

FIG. 3. - Pore génital entouré de fines épines, d'un individu de même provenance que ceux des figures 1 et 2 . 
testicule antérieur et s'étendent jusqu'à l'extrémité postérieure du corps, couvrant les cæca intestinaux. La branche impaire de la vessie excrétrice s'étend jusqu'au bord postérieur du testicule postérieur, où elle se divise.

Les œufs sont peu nombreux, de 7 à 28 , et mesurent $84 \times 58,5,88 \times 62,4$, $94 \times 62,4 \mu$.

\section{Discussion :}

Parmi les 17 espèces admises dans Mesorchis E. Dietz 1909 par K. I. Skrjabin et E. Ya. Baschkirova (1956, p. 701-735, fig. 232-245), aucune n'est mentionnée comme ayant été trouvée chez un Podiceps et une seule présente des caractères suffisamment voisins de ceux de nos spécimens pour que leur conspécificité soit probable, c'est M. pseudoechinatus (P. Olsson 1876). Cette espèce est surtout connue par les descriptions et figures qui en ont été données par P. Olsson (1876, p. 21-22, 34, pl. IV, fig. 45-49) d'après des spécimens du rectum de Larus marinus L. 1758, à Lund (Suède) et par A. Looss (1899, p. 685-686, pl. XXV, fig. 11-12, 15 a) d'après des spécimens de la moitié postérieure de l'intestin d'un Larus fuscus L. 1758 de Basse-Egypte.

Chez les spécimens d'Olsson et de Looss, les vitellogènes atteignent seulement le testicule postérieur, ce qui est typique pour le genre. Cependant, cette limite antérieure des vitellogènes ne paraît pas rigoureusement fixe et Skrjabin et Baschkirova (1956, p. 705, fig. $232 a$ et $b$ ) donnent des figures d'individus rapportés à pseudoechinatus par Baschkirova (1941) et par Oschmarin, chez lesquels les vitellogènes atteignent antérieurement, dans un cas, la mi-longueur du testicule antérieur, dans un autre le bord antérieur du testicule antérieur. Jusqu'à présent, dans un même lot de spécimens récoltés en même temps dans un même individu-hôte, on n'a pas observé à la fois des individus à vitellogènes atteignant seulement le bord postérieur du testicule postérieur et des individus à vitellogènes dépassant le bord postérieur du testicule antérieur. Je considère donc ces derniers comme une variété bien définie, différente de la forme typique pour l'espèce.

Contrairement à ce que l'on a admis pendant longtemps, pseudoechinatus ne se trouve pas seulement chez divers Larus, Rissa tridactyle (L.), Mergus merganser L., il a aussi été identifié chez Podiceps cristatus (L. 1758) et Podiceps griseigena (Boddaert 1783) par plusieurs helminthologistes russes, mentionnés par Irène Bychovskaja-Pavlovskaja (1962, p. 32, 273).

\section{ECHINOCHASMUS (ECHINOCHASMUS) MILITARIS J. - A. Leonov 1958}

(Fig. 4)

\section{Matériel examiné :}

Quatre individus, dont un incomplet et un en mauvais état, de l'intestin d'Egretta alba (L. 1758), Akinlou (Kurdistan), 27-4-1960. 


\section{Description :}

Longueur 1,85 , largeur 0,50 . Ventouse orale longitudinalement 0,055 , transversalement $0,074 \mathrm{~mm}$ (chez un autre individu, diamètre 0,090 ). Ventouse ventrale presque entièrement en avant de la mi-longueur, diamètre 0,436 . Prépharynx, longueur environ 0,04 . Pharynx $0,109 \times 0,058 \mathrm{~mm}$. Epines céphaliques 24 ; les grandes antérieures et latérales $58,5 \times 20 \mu$, la plus interne $54,6 \times 27 \mu$.

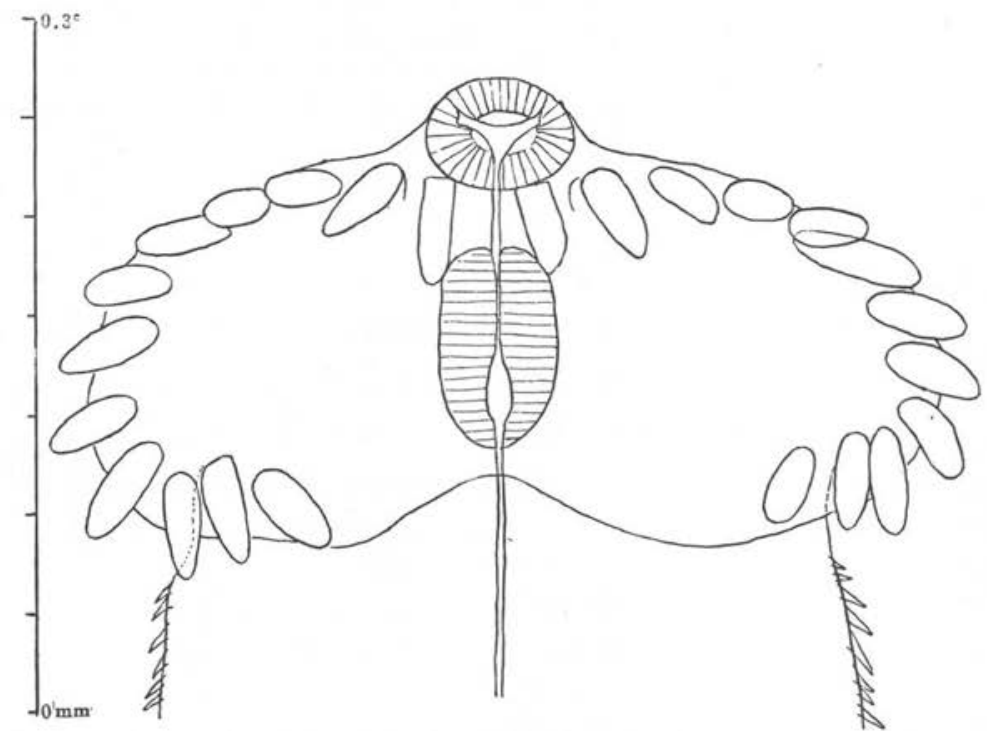

FIG. 4. - Echinochasmus (Echinochasmus) militaris V. A. Leonov 1958 d'Egretin alba (L.), à Akinlou (Kurdistan). Y. Golvan leg., 27.4.1960. Plateau céphalique.

Testicules lobés, l'antérieur $0,335 \times 0,200 \mathrm{~mm}$, le postérieur moins large mais beaucoup plus long $0,265 \times 0,305$. Ovaire globuleux, diamètre 0,107 . Poche du cirre ovale, contre le bord antérieur de la ventouse ventrale ou en partie dorsale à la ventouse ventrale, $0,200 \times 0,098$ à $0,204 \times 0,120$.

Vitellogènes atteignant le niveau du bord postérieur de la ventouse ventrale, confluents en arrière des testicules.

CEufs peu nombreux ( 2 à 6 ), mesurant $66,3 \times 44,70,2 \times 44,80 \times 46,8 \mu$.

\section{Discussion :}

Chez les Ardéidæ paléarctiques sont connues sept espèces d'Echinochasmus (Echinochasmus) à 24 épines céphaliques : beleocephalus (Linstow 1873), euryporus (Looss 1896), militaris V. A. Leonov 1958, amphibolus A. Kotlan 1922, coaxatus E. Dietz 1909, ruficapensis Verma 1935, bagulai Verma 1935. Les trois premières ont été iden- 
tifiées chez Egretta alba (L.), mais la troisième seulement concorde avec nos spécimens iraniens par l'ensemble de ses caracères; nous les considérons donc comme référables à militaris.

Echinochasmus (E.) militaris V. A. Leonov (1958, p. 200-201, fig. 1) a été récolté chez Ardea cinerea L. et Egretta alba (L.) des limons du Dniepr et de Iagorlitzk.

Comme le remarque Leonov, militaris est quelque peu voisin d'E. (E.) bagulai S. C. Verma 1935 (p. 838-843, fig. 1-4) de l'intestin grêle d'Ardeola grayi (Sykes) et de Nycticorax nycticorax (L.) d'Allahabad et Nagpur (Inde) (1) ; il en diffère en particulier par les dimensions presque doubles de sa ventouse ventrale et du plateau céphalique, ses épines céphaliques beaucoup plus grandes, son pharynx aussi beaucoup plus grand, pour une longueur du corps très peu plus grande.

Super-Famille PLAGIORCHIOIDEA $\quad$ R. - Ph. Dollfus 1929

Famille ENCYCLOMETRIDAE K. Odening 1960, p. 448 (1)

Sous-Famille ENCYCLOMETRINAE

(H.-R. Mehra 1931) W. Nicoll 1932

Genre encyclometra H. - A. Baylis et H. - G. Cannon 1924

syn. Odhneria J.-G. Baer 1924, préemployé

Paraplagiorchis R.-Ph. Dollfus 1924

ENCYCLOMETRA COLUBRIMURORUM

(Rudolphi 1819) R. - Ph. Dollfus 1929

(Fig. 5-7)

\section{Matériel de la collection :}

Huit individus, un peu macérés et fortement contractés, de l'estomac de Natrix tessellata Laurenti 1768, Téhéran, 3-5-1959.

(1) Mendheim (1943, p. 282), mentionne Podiceps ruficollis (Pallas) comme hôte de bigulai, mais Verma $(1935$, p. 843$)$ ne le mentionne pas.

(1) K. Odening (1960, p. 448) fonde cette famille principalement sur une disposition particulière des gros canaux collecteurs aboutissant à la vessie excrétrice; de chaque terminaison de la vessie en $Y$ part un gros canal collecteur se dirigeant antérieurement et qui, un peu avant la ventouse orale, se recourbe et se continue en direction postérieure par un unique canal collecteur. Cette disposition est considérée par Odening comme s'opposant à celle existant dans l'ensemble des Plagiorchiidae, où le gros canal collecteur aboutissant à la vessie se bifurque en une branche ascendante et une branche descendante. Cette branche descendante existe seule chez Encyclometra colubrimurorum (Rud.), d'après Odening (1960, p. 447, fig. 2 ).

Déjà P. Sonsino $(1892$, p. 92) avait observé que chacun des gros canaux aboutissant à la vessie s'étendait jusqu'à la ventouse orale où il se recourbait en anse.

H. R. Mehra (1931, p. 46-47, fig. 2) dit que le collecteur principal aboutissant à la vessie s'étend en direction antérieure aussi loin que le niveau du bord antérieur du pharynx, puis se recourbe pour se continuer en direction postérieure jusqu'en arrière de la ventouse ventrale. C'est exactement ce qui a été dit par Odening (1960, p. 448); toutefois, la figure donnée par Mehra montre, 


\section{Description :}

Corps ovale, déprimé, jamais étroit-filiforme, mesurant $2 \times 0,8$ à $3,1 \times 1 \mathrm{~mm}$. La présence d'une protubérance à l'extrémité postérieure du corps est inconstante. Pour un individu long de $3,1 \mathrm{~mm}$, la ventouse orale a un diamètre de 0,57 , la ventrale un diamètre de $0,65 \mathrm{~mm}$, le pharynx mesure $0,38 \times 0,27 \mathrm{~mm}$.

Il n'y a ni prépharynx, ni œsophage, mais il est possible que chez des individus en grande extension, ces organes apparaissent : ils sont présents sur la figure schématique donnée par J.-G. Baer (1924, p. 23, fig. 1). Les cæca intestinaux atteignent presque l'extrémité postérieure du corps, ils sont égaux ou subégaux ; l'inégalité, quand elle existe, est faible (2). Les testicules, toujours en tandem, sont ronds ou quelquefois à bords ondulés ou un peu lobés. La poche du cirre a une grosseur très variable, elle est complètement ou partiellement en avant de la ventouse ventrale et le pore génital est déplacé vers la gauche. L'ovaire est en partie ou complètement dorsal à la ventouse ventrale, rarement complètement en arrière d'elle; il est globuleux ou de forme irrégulière. Les vitellogènes recouvrent les cæca intestinaux, la grosseur des follicules est très variable; ils débutent antérieurement vers le niveau du bord postérieur de la ventouse ventrale, s'étendant jusqu'à l'extrémité du corps, se rejoignant presque toujours par quelques petits follicules.

Les branches de la vessie excrétrice atteignent le niveau de l'ovaire, ou presque, et reçoivent un gros canal collecteur ascendant.

L'utérus est d'abord localisé de part et d'autre des testicules, les branches ascendante et descendante passant entre les testicules et les vitellogènes. Chez les individus âgés, à très nombreux œufs, les sinuosités utérines remplissent tout l'espace compris entre la ventouse ventrale et l'extrémité postérieure du corps, atteignant latéralement les vitellogènes; elles pénètrent dans l'espace intertesticulaire mais ne s'étendent pas ventralement aux testicules. Les œufs en bon état que j'ai mesurés ont, en moyenne, $83,9 \times 43,9 \mu$.

Pour une description plus détaillée de cette espèce, qui présente une grande variabilité, on se reportera aux ouvrages mentionnés ci-après, en particulier à $\mathrm{R}$.-Ph. Dollfus $(1924 a, 1924 b)$.

de chaque côté, aboutissant au point de courbure, trois minces canaux collecteurs qui ne correspondent pas à la description.

Remarquons que le gros canal collecteur ascendant, figuré par S. Yamaguti (1933, p. 81, fig. 33), pour Encyclometra microrchis S. Yamaguti, s'étend de la vessie au niveau du pharynx sans se diviser en branche ascendante et branche descendante.

Ceci ne s'accorde pas avec ce qui a été décrit pour Encyclometra japonica S. Yoshida et Y. Ozaki (1929, p. 240-241, fig. 1) par ces auteurs; ceux-ci ont observé que le gros canal collecteur aboutissant à la vessie se divise au niveau du bord antérieur de la ventouse ventrale en une branche ascendante et une branche descendante, au lieu de se continuer, comme dans la description d'Odening, par une unique branche descendante.

(2) L'inégalité est plus accentuée chez le spécimen-type d'E. subflava (Sonsino) que j'ai figuré en 1924 (p. 574, fig. 1) ; elle est visible aussi sur un des exemplaires provenant de Bologne que j'ai figurés la même année dans une autre publication (1924, p. 271, fig. 5).

J. G. Baer (1924, p. 23) avait déjà constaté qu'un caecum pouvait être plus long que l'autre. 


\section{Discussion :}

La variabilité porte principalement sur la forme générale du corps, le rapport entre le diamètre de la ventouse orale et celui de la ventouse ventrale, la forme globuleuse ou piriforme du pharynx, l'inégalité dans la longueur des cæca intestinaux, la grosseur et la position de la poche du cirre, souvent en partie dorsale à la ventouse ventrale, la grosseur et la position de l'ovaire, souvent entièrement dorsal à la ventouse ventrale, la forme régulière ou non des testicules, leur position en tandem ou oblique, leur distance entre eux et leur éloignement de l'ovaire.

Le caractère de la longueur des cæca a été utilisé par Yeh (1958, p. 100) pour distinguer entre elles les trois espèces qu'il admet dans le genre.

Cæca égaux : colubrimurorum.

Cæca subégaux : japonica.

Cæca très inégaux : asymetrica.

Malheureusement, ce schéma est presque toujours en défaut pour distinguer colubrimurorum de japonica Yoshida et Ozaki, sensu Yeh (1958).

D'après Yeh (1958, p. 111), lorsque les cæca sont inégaux chez colubrimurorum, c'est qu'ils sont « distorted »; s'ils sont égaux chez japonica, c'est par suite de la contraction et lorsqu'il y a contraction, « the cæca become wavy » au lieu d'être rectilignes.

Je ne suis pas d'accord avec Yeh au sujet de l'inégalité des cæca et je figure un spécimen de colubrimurorum d'une Natrix d'Italie, montrant le cæcum droit plus court, sans être « distorted ", et un autre spécimen que je rapporte à la même espèce, d'un Ancistrodon de Chine, ayant aussi le cæcum droit plus court, sans être " distorted ».

\section{Quelques références concernant les diverses appellations d'ENCYCLOMETRA COLUBRIMURORUM (Rudolphi 1819) et sa présence en Europe}

1819. «Distoma Colubri murorum »: C. A. Rudolphi, p. 121 (Musée de Vienne).

1850. « Distomum allostomum Diesing »: C. M. Diesing, p. 367, 658 (nomen novum).

1859. «Distomum caudatum. Polonio »: A. F. Polonio, p. 4.

1881. « Distoma allostomum Diesing »: G. Ercolani, p. 303-305, 309, 332, pl. II, fig. 15 (immature).

1892. «Distomum subflavum, n. sp. » : Pr. Sonsino, p. 91-92.

1893. «Distomum subflavum Sonsino »: Pr. Sonsino, p. 184.

1896. «Distomum (Opisthorchis) caudatum Polonio »: C. Parona, p. 9-11, fig. 4 (syntype de Polonio, musée de Padoue).

1900. «Distomum sp.? n 1 » N. E. Timoféev, p. 151-153, 163, 165, 166, pl. IV, fig. 2. 1924. "Odhneria bolognensis n. gen., n. sp. »: Jean-G. Baer, p. 22-25, fig. 1.

1924. « Encyclometra natricis, gen. et sp. n. »: H. A. Baylis et H. Graham Cannon, p. 194-196, pl. VI. 


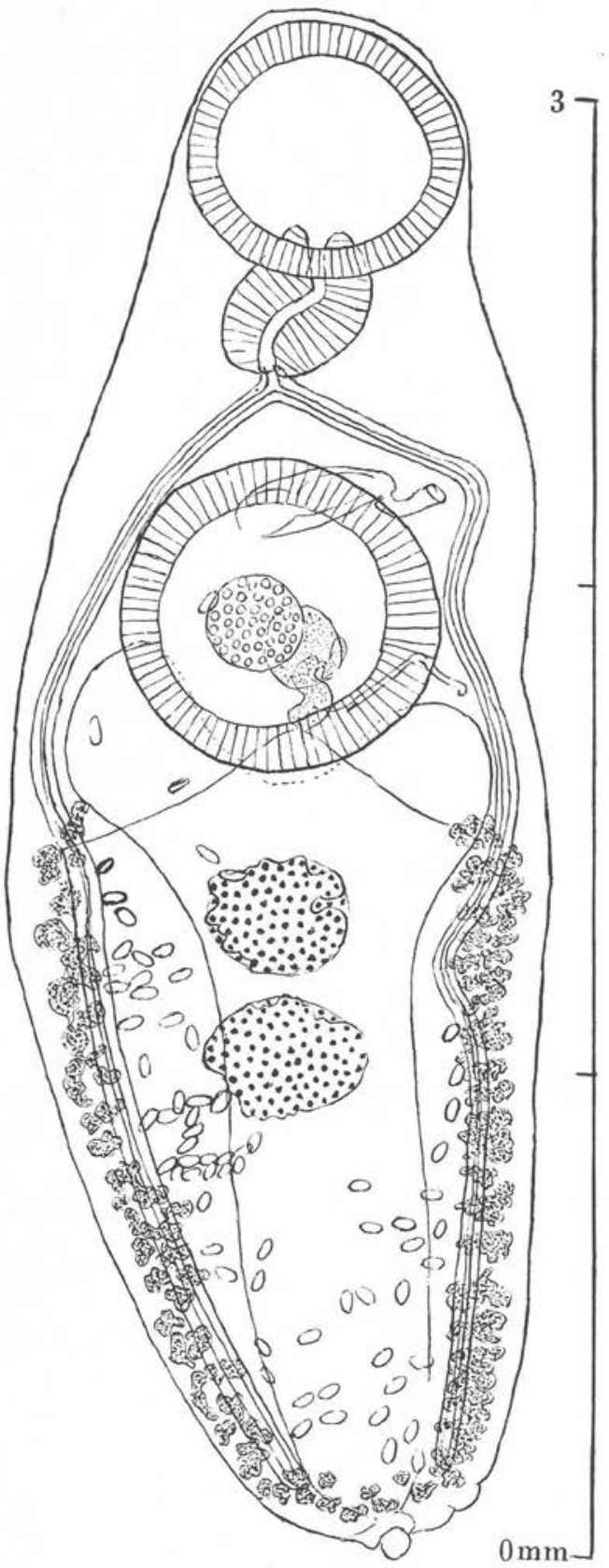

Fic. 5. - Encyclometra colubrimurorum (Rudolphi 1819), de l'estomac de Natrix tessellata Laurenti 1768, Téhéran 3.5.1959. Spécimen fortement contracté, à ovaire dorsal à la ventouse ventrale. $\mathrm{Vu}$ par la face ventrale. 


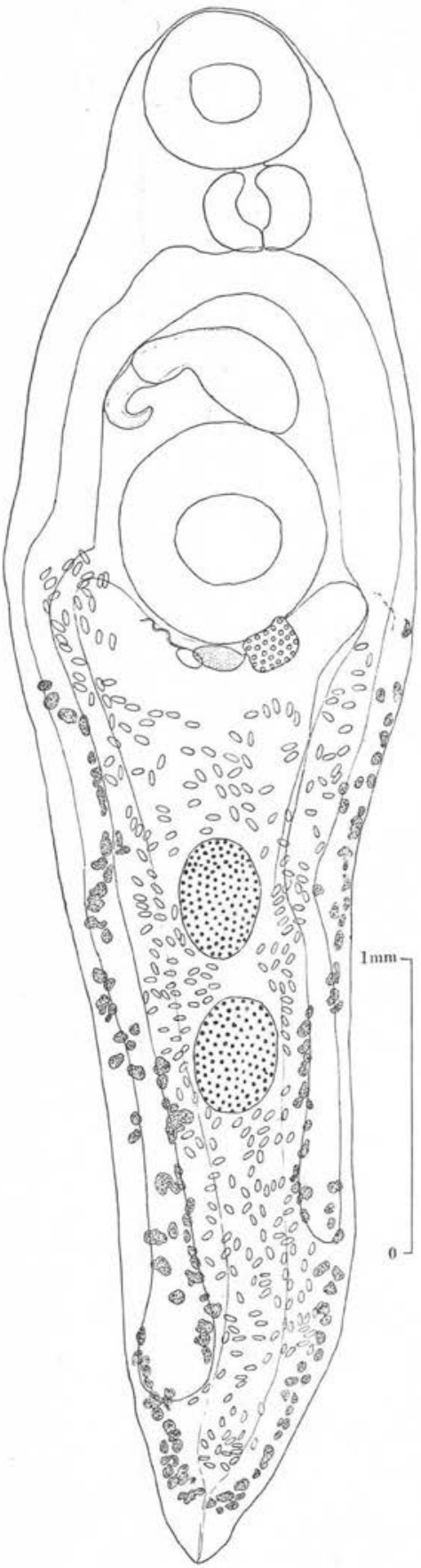

FIG. 6. - Encyclometra colubrimurorum (Rudolphi 1819), de l'estomac de Natrix natrix persa (Pallas 1811), des environs de Bologne (Italie). Dissection au Muséum de Paris. Ipse legi, 10.12. 1924. Spécimen non contracté à caeca inégaux, vu par la face dorsale.

Longueur 5,1 mm, largeur $1,4 \mathrm{~mm}$; ventouse orale $0,523 \times 0,430$; ventouse ventrale $0,720 \times 0,690 ;$ pharynx 0,292 $\times 0,400$; ovaire $0,185 \times 0,185$; testicule antérieur $0,415 \times 0,276$; testicule postérieur $0,415 \times 0,307 \mathrm{~mm}$. 
Fig. 7. - Encyclometra colubrimurorum (Rudolphi 1819) de l'œsophage d'Ancistrodon halys brevicauda L. Stejneger 1907. Chine. Shen Tseng leg., 15.11.1928. Spécimen non contracté, à caeca inégaux, vu par la face dorsale.

Longueur $4 \mathrm{~mm}$, largeur 1,3 ; ventouse orale 0,384 $\times 0,384$, ventouse ventrale $0,461 \times 0,507$; pharynx $0,230 \times 0,215$; poche du cirre $0,415 \times$ 0,170 ; ovaire diamètre 0,155 ; testicule antérieur $0,230 \times 0,292$; testicule postérieur $0,430 \times 0,322$ $\mathrm{mm}$. CEufs $83,3 \times 47,2 \mu$. Chez un autre individu, un peu plus grand, ventouse orale $0,384 \times 0,384$; ventouse ventrale $0,461 \times 0,507$; pharynx $0,245 \times$ 0,215 ; poche du cirre $0,415 \times 0,215$; ovaire 0,170 $\times 0,200$; testicule antérieur $0,307 \times 0,384$; testicule postérieur $0,338 \times 0,338$. Les testicules sont très fortement lobés chez tous les individus. L'ovaire, pour cinq individus de même provenance, est complètement dorsal à la ventouse ventrale chez trois, en partie dorsal chez un, complètement en arrière chez un.

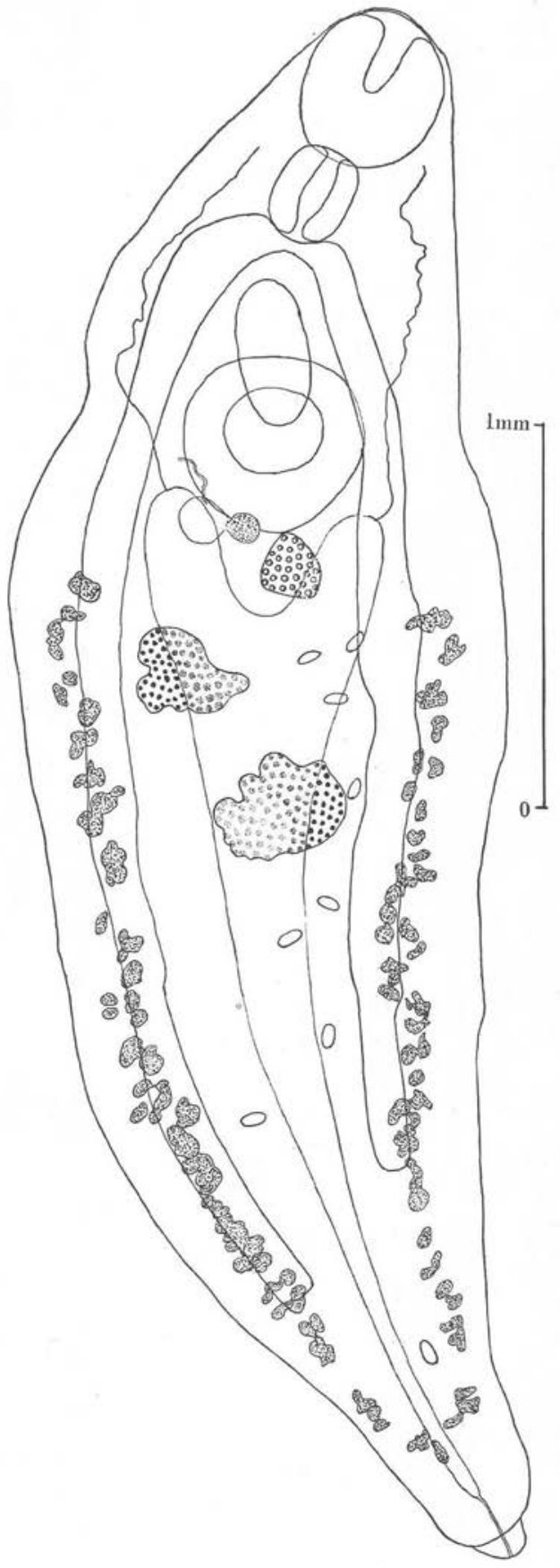




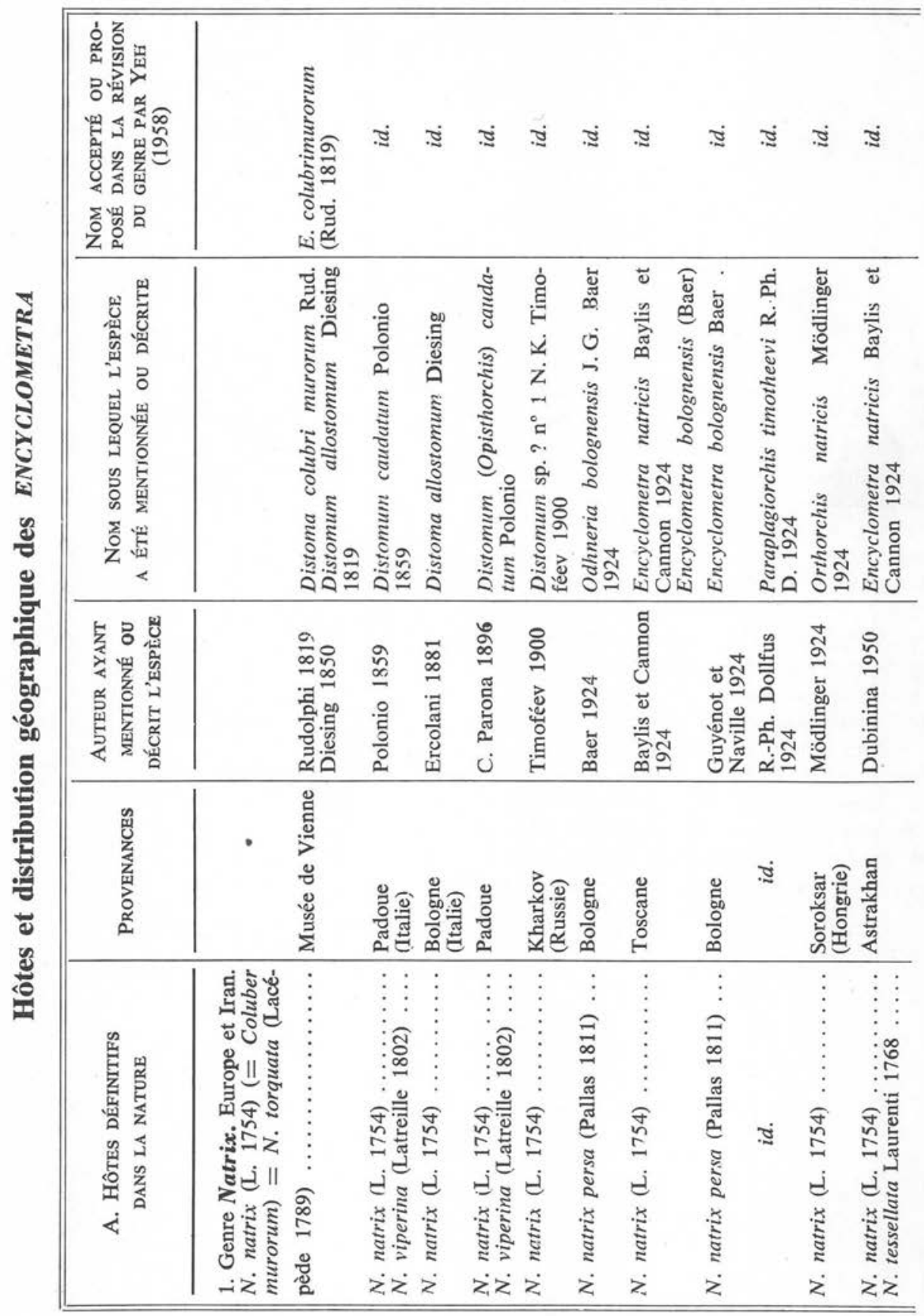




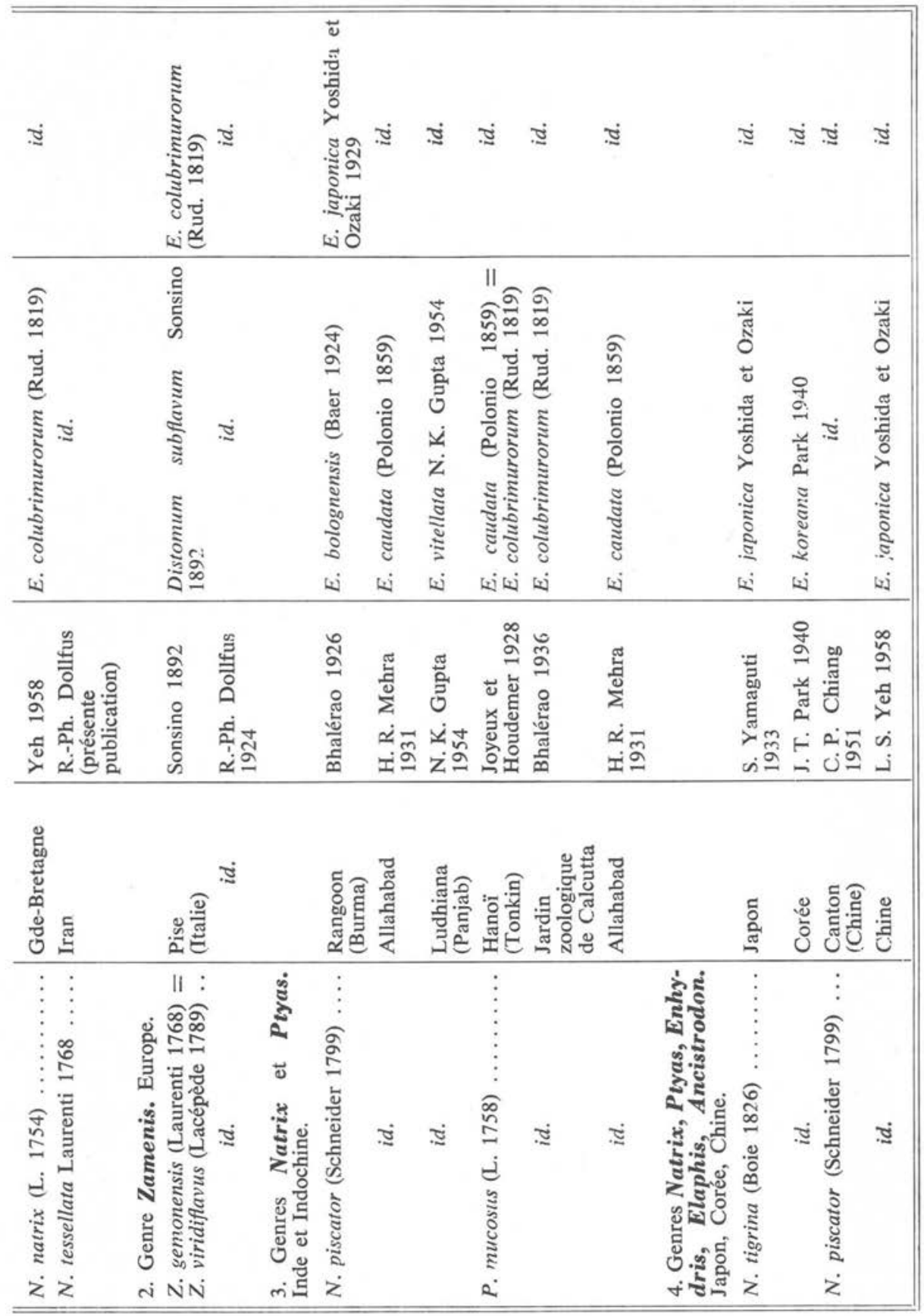




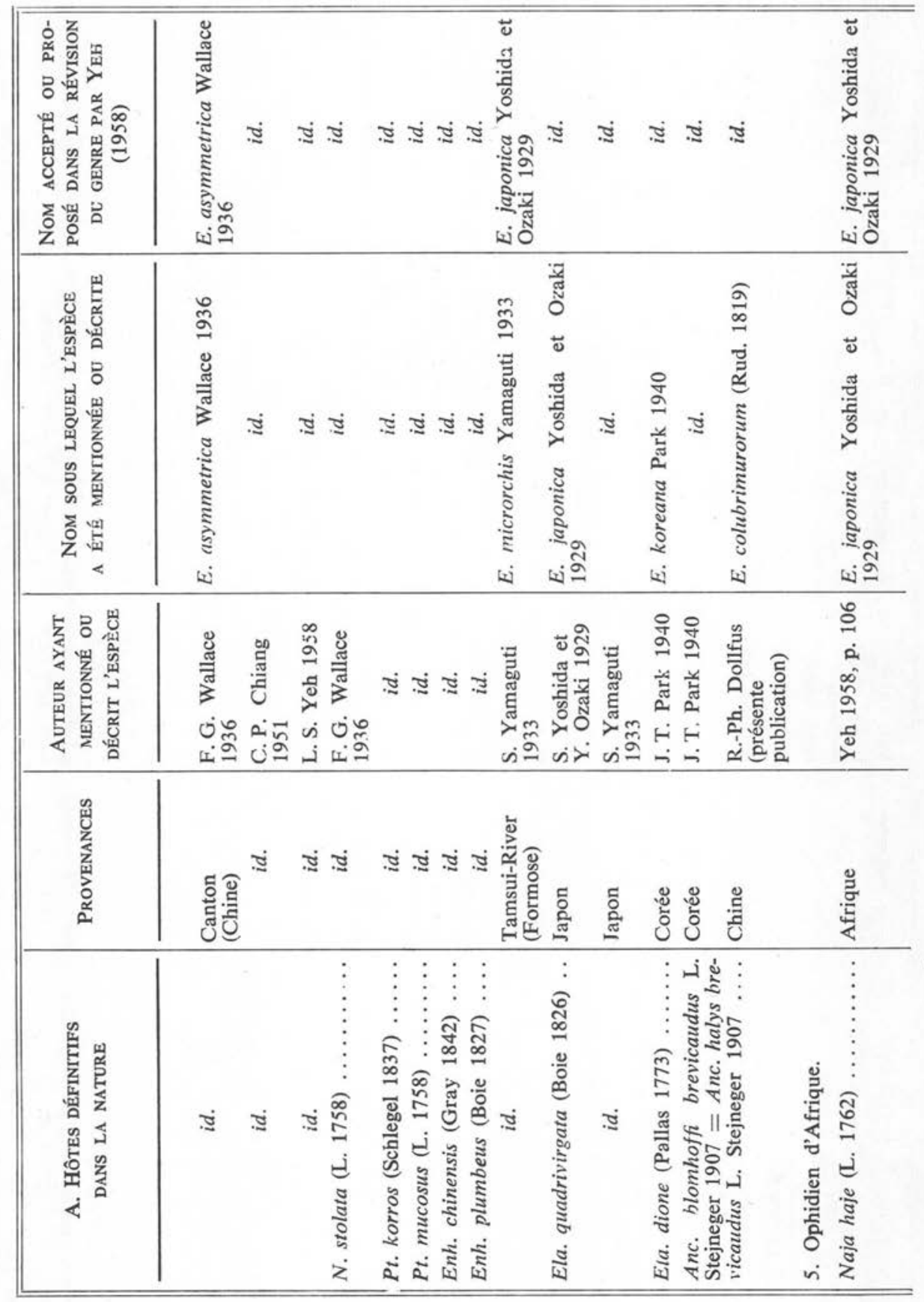




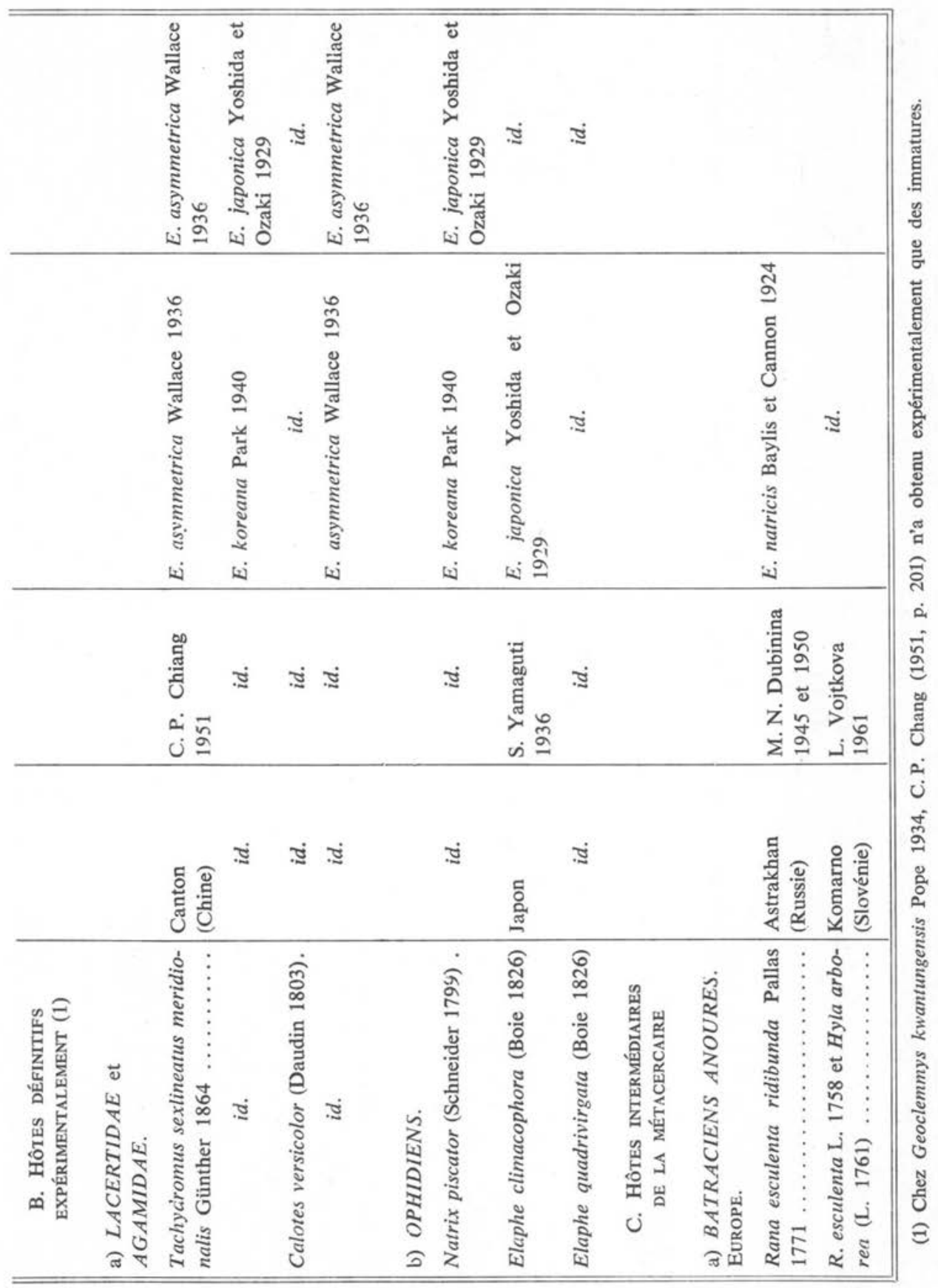


ROBERT-PH. DOLLFUS

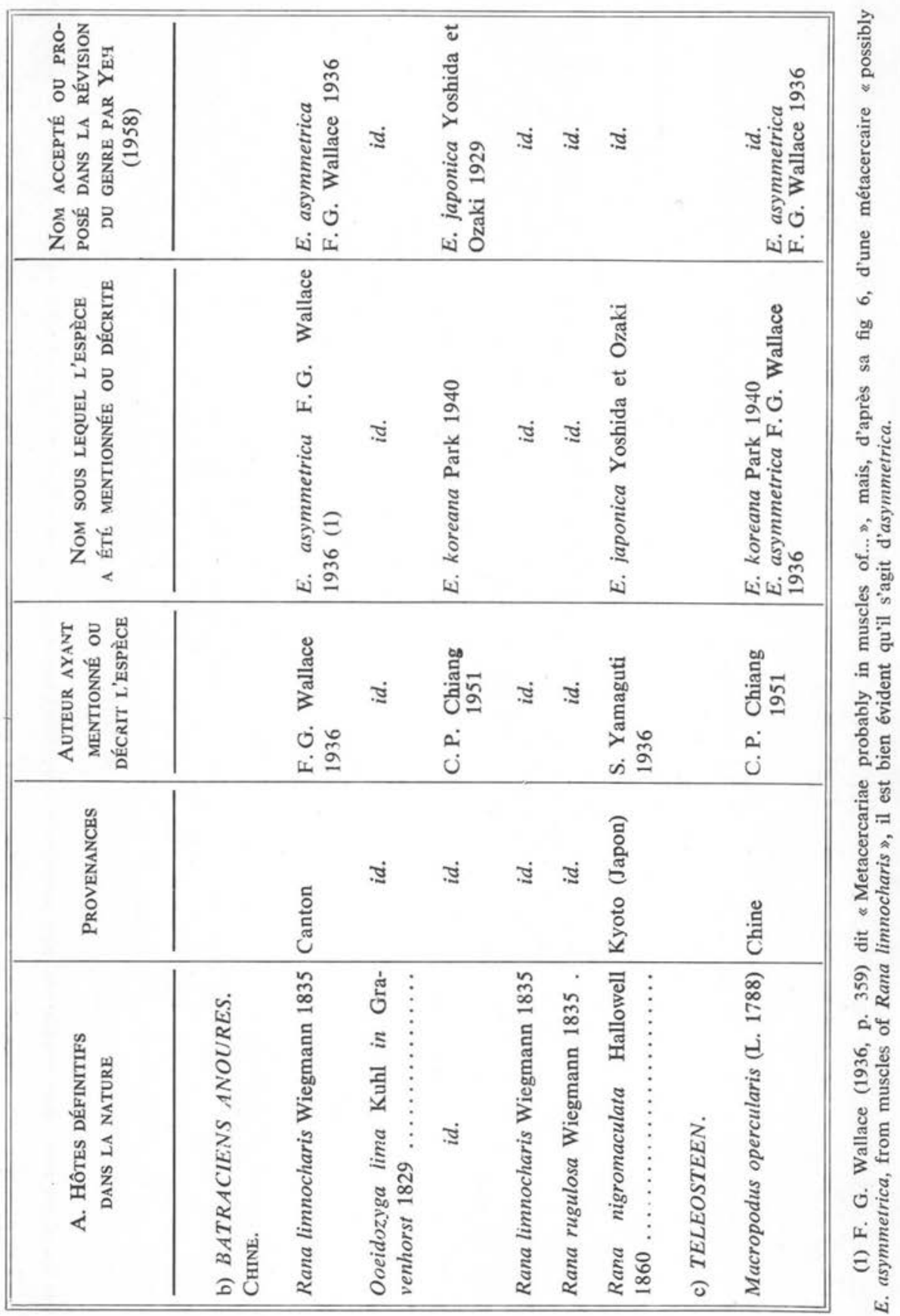


1924. « Encyclometra bolognensis (Baer) » : H. A. Baylis et H. Graham Cannon, p. 558-559. 1924. «ncyclometra bolognensis Baer 》: E. Guyénot et A. Naville, p. 80-83, fig. 1-2. 1924. « Paraplagiorchis timotheevi nom. nov. » : R.-Ph. Dollfus, p. 268-275, fig. 1-8.

1924. « Encyclometra subflava (Sonsino 1892), R.-Ph. Dollfus 1924 » R.-Ph. Dollfus, p. 572-576, fig. 1 .

1924. «Orthorchis natricis nov. gen., n. sp. »: G. Mödlinger, p. 193-195, 198, pl., fig. 5.

1928. «Encyclometra caudata (Polonio, 1859) »: R.-Ph. Dollfus, in Ch. Joyeux et F. E. Houdemer, p. 47-54.

1928. «Encyclometra colubrimurorum (Rud., 1819) »: R.-Ph. Dollfus, in Ch. Joyeux et F. E. Houdemer, p. 47.

1929. « Encyclometra colubrimurorum (Rud., 1819) 》: R.-Ph. Dollfus, p. 34, note 1.

1942. «ncyclometra colubrimurorum (Rudolphi, 1819) Dollfus, in Joyeux et Houdemer 1928 » : R. Chester Hughes, Joë W. Higginbotham et Jasper W. Clary, p. 120 ; 1942, p. 38,39 .

1945. "Encyclometra natricis \&: M. N. Dubinina, p. 168, 169 (larve).

1946. «Encyclometra caudata (Polonio, 1859) Joyeux et Houdemer, 1928 » Ben Dawes, p. 300, fig. 49 A, d'après J. G. Baer, 1924.

1950. «Encyclometra natricis Baylis et Cannon, 1924 » M. N. Dubinina, p. 320, 321, 339, 346. fig. $9 a$, larve, fig. $9 b$, adulte.

1958. « Encyclometra colubrimurorum (Rudolphi, 1819) Dollfus, 1929 » L. S. Yeh, p. 99102, 110, 111-112, fig. 2-4.

1961. «ncyclometra natricis Baylis et Cannon, $1924 »:$ L. Vojtkova, p. 25, 26, 27, 28 (larve).

\section{Quelques références d'ENCYCLOMETRA récoltés aux Indes} et au Tonkin et rapportés à E. COLUBRIMURORUM Rud.

avant qu'une autre espèce ait été décrite dans le genre ou que les Helminthologistes aient pris connaissance de la description d'une autre espèce

1926. «Encyclometra bolognensis (Baer, 1924) 》 : G. D. Bhalérao, p. 4-5.

1927. «ncyclometra caudata (Polonio, 1859) 》: F. E. Houdemer, p. 215.

1928. «ncyclometra caudata (Polonio, 1859) » : R.-Ph. Dollfus, in Ch. Joyeux et F. E. Houdemer, p. $47,54$.

1928. Encyclometra colubrimurorum (Rud., 1819) : R.-Ph. Dollfus, in Ch. Joyeux et F. E. Houdemer, p. 47.

1931. Encyclometra caudata (Polonio, 1859) »: H. R. Mehra, p. 43-47, 52, fig. 2, pl. VI, fig. 6 .

1936 « Encyclometra colubrimurorum (Rud., 1819) Dollfus, 1931 » G. D. Bhalérao, p. 195.

Dans sa révision du genre, Yeh Liang-Shen (1958, p. 104) rapporte à E. japonica S. Yoshida et Y. Ozaki 1929 les spécimens récoltés au Tonkin par E. Houdemer et 
ceux récoltés aux Indes par Bhalerao et Mehra. Je suppose que Yeh se fonde sur la distribution géographique qu'il donne pour japonica, car Joyeux et Houdemer n'ont donné aucune description de leurs spécimens du Tonkin; ils ont seulement accepté l'identification à caudata $=$ colubrimurorum que je leur avais proposée d'après une de leurs préparations. J'ai sous les yeux cette préparation : il s'agit d'un individu à région antérieure fortement rétractée, d'où l'absence de prépharynx et d'œsophage. Il mesure $3,5 \times 1,1 \mathrm{~mm}$, la ventouse orale $0,430 \times 0,446$, la ventrale $0,430 \times 0,538$, le pharynx $0,261 \times 0,245 \mathrm{~mm}$. Le cæcum gauche est plus long que le droit, tout autant que chez le spécimen-type de subflavum que j'ai figuré. Les œufs ont 80,4 × 41,6 $\mu$.

Je ne vois aucune raison pour attribuer ce spécimen du Tonkin à japonica. La description et les figures que Yeh (1958, p. 104-110, fig. 5-9) donne de japonica peuvent aussi bien concerner colubrimurorum.

Bhalerao (1926 et 1936) ne fait aucune allusion à une inégalité des cæca et Mehra (1931, p. 43), qui a observé le cæcum gauche un peu plus long que le droit, a cependant figurè (p. 46, fig. 2) un individu ayant les cæca égaux.

E. japonica S. Yoshida et Y. Ozaki (1929, p. 239-243, fig. texte 1, pl. I, fig. 1-3), si l'on se reporte à la description et aux figures originales, a un habitus et une disposition des organes qui semblent l'éloigner de colubrimurorum : corps élancé, plus large au niveau de la ventouse ventrale, ventouses plus petites, la ventrale très antérieure, très rapprochée de l'orale, ovaire assez loin en arrière de la ventouse ventrale, testicules en oblique. Ces caractères ont-ils une valeur pour la discrimination spécifique ? C'est possible, mais incertain. Remarquons que S. Yoshida et Y. Ozaki n'ont observé aucune différence de longueur entre les cæca et que Yamaguti (1933, p. 80), chez les spécimens qu'il décrit comme japonica, ne fait aucune allusion à une différence de longueur des cæca; il ne s'agit donc pas d'un caractère de discrimination spécifique. Cependant, Yeh (1958, p. 105) dit que, chez japonica, les cæca sont « always unequal with the left caecum slightly longer than the right one ».

En synonymie de japonica, Yeh place E. microrchis Yamaguti 1933, E. koreana Park 1940 et E. vitellata N. K. Gupta 1954.

E. microrchis S. Yamaguti (1933, p. 80-82, fig. 33 ; 1936, p. 570-571, fig. 23), qui a le cæcum droit plus long que le gauche (3), ne diffère pas de l'espèce figurée par Yeh comme japonica. L'ovaire est figuré comme entièrement dorsal à la ventouse ventrale. mais ce n'est pas un caractère distinctif.

E. Koreana J. T. Park (1940, p. 113-117, fig. texte 1-2, pl. XI, fig. 1-4) mesure 1,8-6,7 $\times$ 0,65-1,75 mm, a les cæca tantôt symétriques, tantôt asymétriques. Park a insisté sur les variations individuelles très étendues. La vésicule séminale, dit Park, est un court tube recourbé ou sinueux, alors qu'elle est un simple sac rectiligne chez japonica. Il est, selon moi, douteux que ce caractère soit constant et discriminatif. La description et les figures données par Park pourraient tout aussi bien être rapportées à colubrimurorum.

(3) La fig. 33 de Yamaguti 1933 est indiquée comme une vue ventrale, mais il semble plutôt que ce soit une vue dorsale; si c'était une vue ventrale, le caecum droit serait plus court que le gauche, alors que la description précise que le droit est plus long. La description de 1933 concerne un unique specimen mesurant $3,6 \times 0,81 \mathrm{~mm}$. 
E. vitellata N. K. Gupta (1954, p. 139-141, fig. 1), qui atteint presque $9 \times 2,5 \mathrm{~mm}$, a le cæcum gauche toujours plus long que le droit. Pour Yeh (1958, p. 105-108), c'est la même espèce que japonica, mais j’estime plus justifié de placer vitellata en synonymie d'asymmetrica (4).

Une espèce bien différente de colubrimurorum est $E$. asymmetrica F. C. Wallace (1936, p. 357-362, fig. 4 et 7, adultes à maturité, fig. 5, adulte immature, fig. 6, métacercaire). Le corps est beaucoup plus élancé $(3,2 \times 1,5$ à 7,16 $\times 0,9 \mathrm{~mm})$ que celui des plus étroits colubrimurorum et le cæcum gauche, chez tous les individus, est toujours tellement plus court que le droit que cette inégalité n'est pas comparable à celle que l'on peut observer chez colubrimurorum et chez les spécimens figurés comme japonica par Yeh. Une bonne figure d'asymmetrica a été donnée par Yeh (1958, p. 101, fig. 1) et j'ai sous les yeux un syntype que je dois à l'amabilité de F. G. Wallace, me permettant d'être pleinement d'accord avec Yeh pour admettre asymmetrica comme espèce indépendante et bien caractérisée.

\section{Famille PLAGIORCHIIDAE (Max Lühe 1901) \\ Sous-Famille SAPHEDERINAE (J.-G. Baer 1924) \\ SAPHEDERA A. Looss 1902}

= Macrodera A. Looss 1899 [non Macroderes Westwood 1843, Coléoptère] (1)

\section{SAPHEDERA LONGICOLLIS (P.-C. Abildgaard 1806)}

Syn. : Fasciola longicollis P. C. Abildgaard in O. F. Mueller 1806 (2),

Distoma naja Rudolphi 1819 ,

Macrodera naja A. Looss 1899 ,

Saphedera naja A. Looss 1902,

Macrodera longicollis (Abildgaard) Max Lühe 1909.

\section{Matériel de la collection :}

Un spécimen (coloré au carmin et monté dans du baume du Canada) du poumon de Natrix tessellata Laurenti 1768, Téhéran, 3 mai 1959.

Ce spécimen est conforme aux descriptions classiques de l'espèce.

(4) N. K. Gupta essaye de justifier la création de vitellata comme espèce nouvelle par la présence d'un vitelloducte transverse additionnel près de l'extrémité postérieure du corps. En réalité, c'est tout simplement la liaison habituelle, plus ou moins visible selon les individus, entre les vitellogènes droit et gauche.

(1) Le Code de la Nomenclature Zoologique (1961, p. 104) recommande de ne pas publier un nom de genre différant d'un autre seulement par sa terminaison. Si on n'acceptait pas cette recommandation, il y aurait plusieurs familles et plusieurs sous-familles du même nom.

(2) L'espèce a été décrite et figurée par Abildgaard (p. 34-35, pl. CLI, fig. A 1-2) dans le vol. IV de Zoologia Danica, qui a été publié en 1806. Il n'en est pas question dans les volumes précédents. Les vol. I et II ( $2^{\circ}$ édition) ont paru en 1788 , le vol. III en 1789. 


\section{Famille PLAGIORCHIIDAE (Max Lühe 1901) Sous-Famille opISTHIOGLYPHINAE R.-Ph. Dollfus 1949 OPISTHIOGLYPHE ENDOLOBA (F. Dujardin 1845)}

(Fig. 8)

\section{Matériel de la collection :}

Environ 24 individus, de l'intestin moyen de Rana esculenta ridibunda Pallas, à Akinlou (Kurdistan), 12-6-1960.

\section{Description :}

Ces spécimens mesurent, par exemple : $1,320 \times 0,484,1,430 \times 0,484,1,826 \times$ $0,572 \mathrm{~mm}$.

Pour un spécimen mesurant $1,476 \times 0,528$, on a $(\mathrm{mm})$ :

\begin{tabular}{|c|c|}
\hline Ventouse orale $\ldots \ldots \ldots \ldots \ldots \ldots \ldots \ldots$ & 0,124 \\
\hline Ventouse ventrale $\ldots \ldots \ldots \ldots \ldots \ldots$ & 0,100 \\
\hline Prépharynx $\ldots \ldots \ldots \ldots \ldots \ldots \ldots \ldots \ldots$ & 0,040 \\
\hline Pharynx ..... & $0,0546 \times 0,0858$ \\
\hline
\end{tabular}

Les testicules sont en tandem, un peu plus larges que longs, comme cela arrive souvent, mais non pas étirés transversalement comme chez Op. ranae (Frölich 1791), tel qu'il a été décrit sous le nom de Dist. endolobum Duj. par A. Looss (1894, pl. II, fig. 27) et sous le nom d'Opisthioglyphe ranae (Fröl.) par Max Lühe (1909, p. 107, fig. 84).

Je renvoie aux descriptions que j'ai données antérieurement d'endolobum (Duj.) (voir R.-Ph. Dollfus 1958, p. 569-575, fig. 5-6; 1958, p. 218-225, fig. 4-6; 1960, p. 114).

Je rappelle que la spinulation cuticulaire atteint au plus le niveau du testicule postérieur, alors que chez l'espèce décrite par Looss, elle dépasse le niveau de la terminaison des cæca.

Ainsi que je l'ai exposé dans plusieurs de mes publications antérieures, je considère les espèces ranae et endoloba comme distinctes et je rapporte à endoloba les spécimens iraniens ci-dessus décrits.

Klaus Odening (1960, p. 342-346, fig. $3 a-b$; 1961, p. 192, 197-201, fig. 18-27) n'est pas d'accord et attribue à des variations individuelles intraspécifiques les différences que j'ai énumérées pour séparer les deux espèces. Il semble en effet qu'il y ait des individus d'endoloba atypiques, présentant des caractères de ranae, à côté d'individus typiques de ranae. Par exemple, le spécimen de la fig. 27 d'Odening (1961, p. 201) correspond bien à ranae, mais je ne peux admettre dans ranae des spécimens tels que 


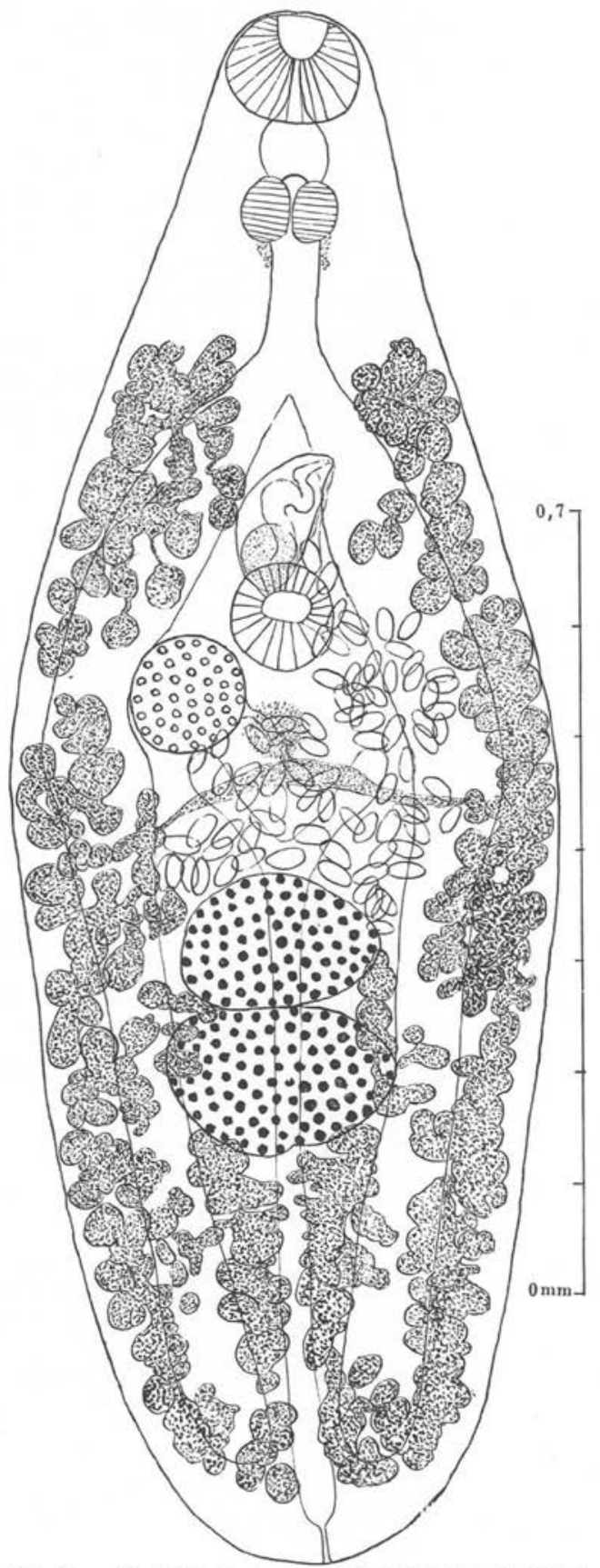

FIG. 8. - Opisthioglyphe endoloba (F. Dujardin 1845), de l'intestin de Rana esculenta ridibunda Pallas, à Akinlou (Kurdistan). Yves Golvan leg., 12-6-1960. 
celui de la fig. 3 d'Odening (1960, p. 342) qui est typiquement endoloba. A mon avis, malgré l'existence de spécimens paraissant intermédiaires, les deux espèces sont différentes.

Famille PLAGIORCHIIDAE (Max Lühe 1901) H.-B. Ward 1917 emend. Sous-Famille PLAGIORCHIINAE (Max Lühe 1901) H.-S. Pratt 1902 emend.

Genre PLAGIORCHIS Max Lühe 1899 =LEPODERMA A. Looss 1899 Sous-Genre MULTIGLANDULARIS R.-Ed.-S. Schulz et A.-A. Skvorzov 1931

PLAGIORCHIS (MULTIGLANDULARIS) BRAUNI

B.-G. Massino 1927

(Fig. 9)

\section{Matériel de la collection :}

Deux individus (colorés au carmin et montés dans le baume du Canada), de l'intestin de Falco (Hypothriorchis) subbuteo L. 1758, à Akinlou, 12-10-1959.

Ces spécimens sont suffisamment voisins, malgré l'absence de prépharynx, de la description originale par B. G. Massino (1927, p. 109-111, fig. 3 ; 1929, p. 135-136, fig. 5), d'après des spécimens récoltés chez Coloeus monedula turrium (Brehm 1831) de la région du Don, pour que l'on puisse les attribuer à cette espèce.

$P$. brauni Massino est admis comme espèce indépendante par I. BychovskaïaPavlovskaïa (1962, p. 77) avec pour synonymes : loossi Massino 1927, massino Petrov et Tichonov 1927, uhlwormi Massino 1927, casarcii Mehra 1937. Cette synonymie a pour conséquence que brauni est cité par Bychovskaïa-Pavlovskaïa (1962, p. 259-260) comme parasitant 31 espèces d'oiseaux (dont Falco subbuteo L.) et le chien, en Russie.

Pour K. Odening (1959, p. 18) et O. E. Fedorova (1954), brauni est un synonyme de P. (Multiglandularis) cirratus (Rudolphi 1802).

J'ai rappelé (1960, p. 288-289) cette synonymie à propos d'une étude sur cirratus et je ne crois pas utile d'en reprendre ici la discussion.

\section{Famille TELORCHIDAE W. Nicoll 1924 \\ Sous-Famille TELORCHIINAE A. Looss 1899 \\ TELORCHIS SOLIVAGUS T. Odhner 1902}

\section{Matériel de la collection :}

Très nombreux individus de l'intestin moyen d'Emys orbicularis (Linné 1758), à Kamalabad, 22-6-1959. 
Fig. 9. - Plagiorchis (Multiglandularis) brauni B. G. Massino 1927, de Falco (Hypothriorchis) subbuteo L. 1758, à Akinlou, 12 octobre 1959. $\mathrm{Vu}$ par la face dorsale. Longueur 2,15, largeur 0,63 , ventouse orale 0,02 , ventouse ventrale, 0,125 , pharynx $0,112 \mathrm{~mm}$. CEufs $39 \times 17-19$ à $41 \times 16 \mu$.

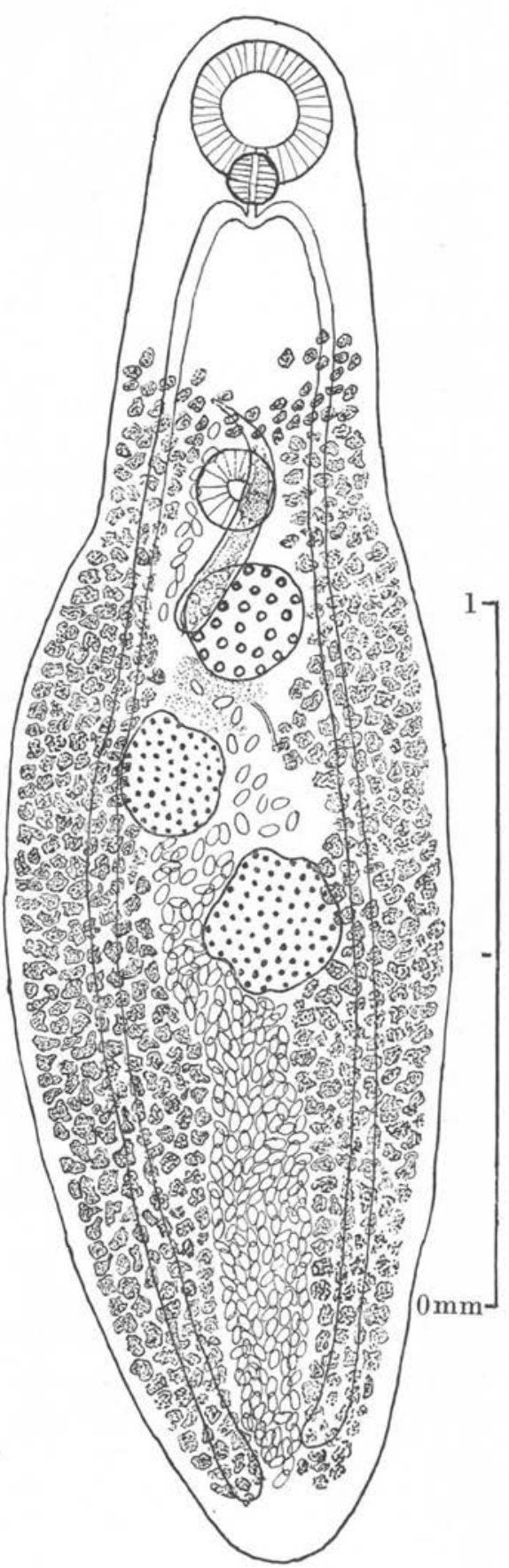




\section{Description :}

J'estime inutile de décrire et figurer une fois de plus cette espèce, je renvoie aux descriptions et figures données par T. Odhner (1902, p. 29-32, fig. 2), K. I. Skrjabine (1924, p. 37-40, fig. 2), Skrjabine (1925, p. 284-289, fig. 2) (1), R.-Ph. Dollfus (1929, p. 45-54, fig. 9-17), Ch. Joyeux et J. Gaud (1945, p. 115-118), etc...

Entre les spécimens des diverses provenances, il y a de petites différences, c'est pourquoi j'avais séparé dans une sous-espèce maroccanus des spécimens récoltés au Maroc.

Je n'ai rien à ajouter à ce qui a été publié par les auteurs ci-dessus cités. Je me contente de remarquer que la différence de diamètre entre la ventouse orale et la ventouse ventrale est inconstante. Chez les petits individus, il arrive que la ventouse orale soit nettement plus grande que la ventrale, mais c'est quelquefois le contraire. Chez les grands individus, c'est presque toujours, mais pas toujours, la ventouse ventrale qui est plus grande que l'orale et, chez le plus grand nombre des individus de taille moyenne, on peut considérer que les ventouses sont subégales. Dans les clefs de détermination, il ne faut donc pas s'appuyer sur une faible différence de diamètre entre les ventouses pour rejeter ou accepter l'identification à solivaqus.

Un autre caractère est quelque peu variable, celui de la longueur des vitellogènes ; chez les individus que j'ai examinés, ils débutent au même niveau, ou presque, à droite et à gauche, mais ils se terminent du côté droit avant ceux du côté gauche.

\section{Dimensions (mm.)}

\begin{tabular}{|c|c|c|}
\hline Longueur ... & 11,8 & 4,0 \\
\hline 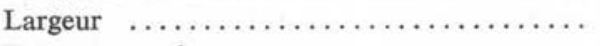 & 0,68 & 0,38 \\
\hline Ventouse orale $\ldots \ldots \ldots \ldots \ldots \ldots \ldots$ & 0,167 & 0,145 \\
\hline Ventouse ventrale $\ldots \ldots \ldots \ldots \ldots \ldots \ldots$ & 0,190 & 0,138 \\
\hline 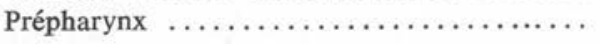 & 0,31 & 0 \\
\hline 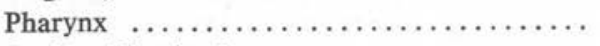 & 0,115 & 0,092 \\
\hline Ovaire (diamètre) $\ldots \ldots \ldots \ldots \ldots \ldots \ldots$ & 0,305 & 0,154 \\
\hline Testicule antérieur longit. . ............ & 0,638 & 0,265 \\
\hline Testicule antérieur transv. $\ldots \ldots \ldots \ldots \ldots$ & 0,457 & 0,265 \\
\hline Testicule postérieur longit. $\ldots \ldots \ldots \ldots \ldots \ldots$ & 0,668 & 0,250 \\
\hline Testicule postérieur transv. . . . . . . . . . & 0,457 & 0,305 \\
\hline Eufs (moyenne) $\ldots \ldots \ldots \ldots \ldots \ldots \ldots \ldots \ldots$ & $31 \times$ & \\
\hline
\end{tabular}

\section{Hôtes et distribution géographique :}

L'espèce est connue de : Clemmys caspica (Gmel.), de Transcaucasie (T. Odhner, 1902) ; Clemmys leprosa (Schweigger), du Maroc (R.-Ph. Dollfus, 1929 ; Ch. Joyeux

(1) Dans la note de Skrjabine, il y a, p. 289, une erreur typographique dont je suis responsable, ayant corrigé les épreuves. Le renvoi à la note infrapaginale doit être placé à la fin de la ligne 5 et non pas à la fin de la ligne 12 . 
et J. Gaud, 1945) (15) ; Emys orbicularis (L.), d'Arménie russe [Skrjabine et Popov, 1924, p. 61 (2) ; Skrjabine, 1924, p. 11 (2) ; 1927, p. 136 (2) ; Skrjabine, 1924, p. 37 ; 1925, p. 185 ; B.-G. Massino, 1924, p. 11] ; de France (J. Poirier, 1886) (3) ; de Sardaigne (M. Stossich, 1904) (3) ; de Hongrie (B. Edélényi, 1962, p. 572-574, fig. 9-10) (4).

\section{TELORCHIS sp.}

(Fig. 10)

\section{Matériel de la collection :}

Environ 40 individus de l'intestin d'une couleuvre, Natrix natrix persa (Pallas), Kamalabad, 22-6-1959.

\section{Dimensions de quatre individus (mm.)}

$\begin{array}{lcccc}\text { Longueur } \ldots \ldots & 1,870 & 2,288 & 2,354 & 2,376 \\ \text { Largeur ...... } & 0,198 & 0,270 & 0,264 & 0,264 \\ \text { Ventouse orale . } & 0,0858 \times 0,0984 & 0,113 & 0,1014 & 0,112 \\ \text { Ventouse } & & & & \\ \text { ventrale } \ldots \ldots . & 0,0702 \times 0,0624 & 0,079 & 0,0585 & 0,080 \\ \text { Pharynx } \ldots \ldots . & 0,0487 \times 0,0,624 & 0,0585 \times 0,0663 & 0,0585 \times 0,080 & 0,080 \times 0,080\end{array}$

(1) Telorchis gabesensis J.S. Ruskowski (1926 p. 327-329, fig. 1) de l'intestin de Clemmys leprosa Schweigger, de l'oasis de Gabès (Tunisie), a la ventouse buccale beaucoup plus grande que la ventrale et une telle différence n'est jamais atteinte chez solivagus; en outre, la spinulation cuticulaire s'étend jusqu'à l'extrémité postérieure du corps, ce qui n'a pas lieu chez solivagus où la spinulation cesse à un niveau un peu variable, mais n'existe plus en arrière des testicules.

(2) Sous le nom de Cercorchis shelkownikowi Skrjabine et Popov 1924.

(3) Distomum poirieri Stossich (1895 p. 227) est le nom proposé par M. Stossich pour désigner le Distome décrit sous le nom erroné de «Distomum gelatinosum, Rud. » par Justin Poirier (1886 p. 33-34, pl. III, fig. 6), qui le trouva, en France vraisemblablement, dans l'intestin d'une Cistude d'Europe, c'est-à-dire Emys orbicularis (L.). C'est presque certainement la même espèce que Stossich (1904 p. 3-5, 8, pl. II fig. 2) a décrite sous le nom de "Telorchis poirieri (Stossich)" de l'intestin du même hôte à Sassari (Sardaigne), malgré une longueur des caeca plus grande que ne l'avait signalé Poirier.

Le seul caractère sur lequel on a essayé de s'appuyer pour considérer l'espèce de Poirier comme distincte de Telorchis solivagus T. Odhner 1902, est une longueur un peu moindre des caeca intestinaux qui, d'après la figure donnée par Poirier, ne dépassent pas le niveau du bord antérieur du testicule postérieur.

Même si l'on suppose exact le niveau de la terminaison des caeca sur la figure de Poirier, il semble bien que cela est seulement un caractère individuel.

T. Odhner (1902 p. 32) indique, comme caractère permettant de distinguer solivagus et poirieri : un rapport différant des dimensions des ventouses, des vitellogènes plus développés, de plus longs caeca intestinaux. A mon avis, ces caractères ne sont pas discriminatifs et lorsque l'on examine de nombreux spécimens de Telorchis, on constate des différences individuelles qui retirent toute valeur à celles admises comme spécifiques par Odhner.

Max Lühe (1909 p. 51-52) qui a accepté poirieri (en le plaçant dans Cercorchis) comme espèce valable, donne comme caractéristique de l'espèce la terminaison des caeca entre les testicules et la terminaison antérieure des vitelogènes un peu en avant de l'ovairc, caractères trop variables pour être discriminatifs.

(4) Sous le nom «Cercorchis Poirieri (Stossich 1895)». Pour l'identification de ses spécimens, Edélényi les a comparés aux données de Lühe (1909), mais la figure 9 d'Edélényi montre un individu ayant les caeca dépassant le testicule postérieur, ce qui est normal, mais en contradiction avec le caractère admis par Lühe comme discriminatif pour poirieri, c'est-à-dire : caeca ne dépassant pas l'espace intertesticulaire. 


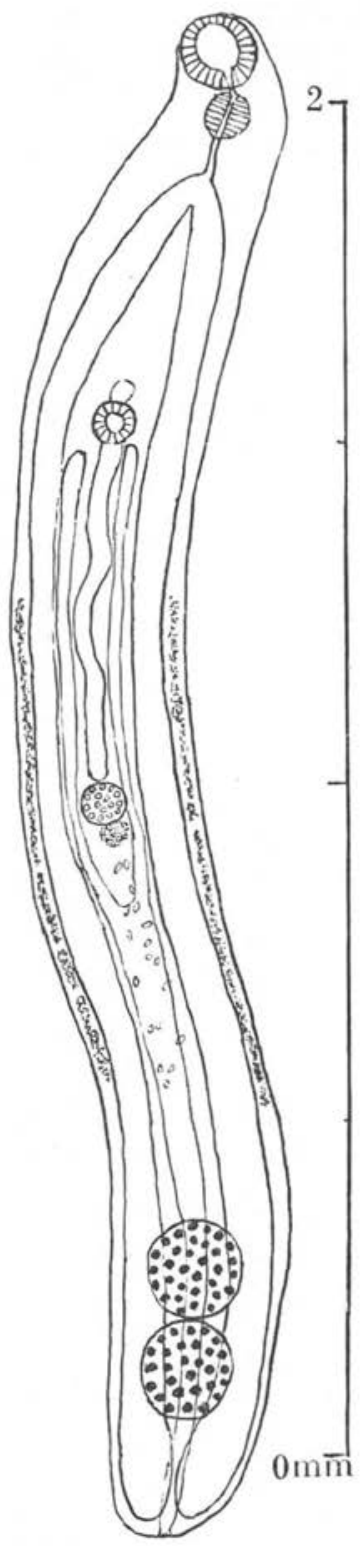

FIG. 10. - Telorchis sp., juv. mesurant $2,288 \times 0,270$. Ovaire, diamètre 0,0471 . Testicule antérieur $0,156 \times 0,147$. Testicule postérieur $0,152 \times$ 0,136 . Le centre de la ventouse orale est à $0,59 \mathrm{~mm}$ de l'extrémité antérieure. Dans l'utérus, il n'y a que queiques œufs mal formés. La spinulation cuticulaire n'est pas figurée.

\section{Description :}

Corps très grêle, subfiliforme. Tous les individus sont jeunes, la plupart immatures ; beaucoup n'ont pas encore de vitellogènes. Cuticule finement spinulée antérieurement ; 
la spinulation disparaît avant le niveau de l'ovaire. Ventouse orale nettement plus grande que la ventrale chez tous les individus. Prépharynx nul ou extrêmement court. Pharynx globuleux ou un peu plus large que long. Esophage une à deux fois aussi long que le pharynx. Cæca intestinaux atteignant l'extrémité postérieure du corps. Testicules ovales longitudinalement ou ronds, subégaux, se touchant. Poche du cirre atteignant le milieu de l'ovaire ou son bord antérieur. Ovaire sur la ligne médiane, un peu en arrière de la mi-longueur du corps. Chez les individus où les vitellogènes sont déjà un peu développés, ils s'étendent sur environ un tiers de la longueur du corps et il n'y a pas de groupes distincts de follicules. Quelques individus seulement ont des œufs, mais en petit nombre et en mauvais état, ils mesurent $31,2 \times 15,6 \mu$.

\section{Discussion :}

Ce qui caractérise ces individus, c'est que leur ventouse orale est nettement bien plus grande que la ventrale et que la largeur de leur pharynx est la même ou peu inférieure à celle de leur ventouse ventrale, alors que, chez assula, quand les ventouses ne sont pas égales, c'est généralement la ventrale qui est la plus grande. C'est une erreur d'indiquer parmi les caractéristiques de l'espèce qu'une des ventouses est plus grande que l'autre et, pourtant, Ben Dawes (1946, p. 302) dit: ventouse ventrale plus petite que l'orale (1), et il adopte pour l'espèce l'appellation « Cercorchis nematoides (Mühling) », qui est à proscrire. Je rappelle que les caractères sur lesquels repose la distinction entre nematoides et ercolanii (2) sont individuels et que cette espèce doit porter le nom de Telorchis assula (F. Dujardin 1845) R.-Ph. Dollfus 1957. Le caractère prétendu distinctif de la poche du cirre, dépassant l'ovaire, est souvent en défaut et il est facile de prendre, pour un dépassement postovarien de la poche du cirre, la glande de Mehlis et le réservoir vitellin, situés dans le prolongement de la poche du cirre, immédiatement en arrière de l'ovaire.

Quoi qu'il en soit, notre Telorchis de Natrix persa (Pallas) d'Iran, malgré ses faibles dimensions et son habitus grêle, ressemble beaucoup à assula jeune, avant la maturité sexuelle ou au début de celle-ci, et il s'agit peut-être de cette espèce ; néanmoins, étant dans l'incertitude, nous ne proposons pas d'attribution spécifique.

(1) Ben Dawes a suivi en cela la description originale de «Distomum nematoides» P. Mühling $(1898$ p. 93 , pl. IV fig. 22) où il est dit que la ventouse orale est toujours plus grande que la ventrale, alors que, le plus souvent, c'est le contraire.

(2) Plusieurs auteurs ont été dans l'incertitude au sujet de la synonymie d'ercolanii et nematoides. Max Lühe (1906 p. 136) a d'abord placé nematoides en synonymie d'ercolanii, mais ensuite (1909 p. 51), il a accepté nematoides comme espèce valable sans citer ercolanii comme synonyme. 


\section{Famille GORGODERIDAE A. Loos 1901 Sous-Famille GORGODERININAE (PHYLLODISTOMATINAE Pigulevsky 1952, (partim) GORGODERINA (GORGODERINA) VITELLILOBA (P. Olsson 1876) Sinitzin 1905}

\section{Matériel de la collection :}

Deux spécimens (colorés au carmin et montés dans du baume du Canada), de la vessie de Rana esculenta ridibunda Pallas 1771, Kamalabad, 22 juin 1959.

Ces spécimens sont conformes aux descriptions classiques de l'espèce.

\section{Famille STRIGEIDAE A. Railliet 1919 Sous-Famille STRIGEINAE A. Railliet 1919 Genre STRIGEA Abildgaard 1790 STRIGEA FALCONIS Viborg 1795-L. Szidat 1928}

\section{Matériel de la collection :}

Environ 135 individus, de l'intestin d'un Milvus migrans (Boddaert 1783), à Bendar-Pahlevi, 14 décembre 1959.

\section{Description :}

Un individu moyen, vu de profil, a une longueur totale de $2,53 \mathrm{~mm}$, la partie antérieure étant un peu inclinée sur la postérieure. En supposant cet individu rectiligne, sa longueur devient $2,882 \mathrm{~mm}$; longueur de la partie antérieure : 0,924 , épaisseur : 1,210 ; longueur de la partie postérieure : 1,958 , épaisseur : $1,056 \mathrm{~mm}$. Ventouse orale, diamètre : 0,167 ; pharynx, diamètre : 0,114 ; ventouse ventrale : $0,182 \mathrm{~mm}$.

Un autre individu, vu de face, est long de $2,772 \mathrm{~mm}$; la partie antérieure mesure 0,902 de long sur 1,254 de large ; la partie postérieure : 1,870 de long sur 1,276 de large. Les œufs, en très bon état, mesurent jusqu'à $104 \times 74 \mu(104 \times 62,4 ; 104 \times 66,3$; $104 \times 70,2)$; de plus petits mesurent $100 \times 54,6 ; 93,6 \times 66,3 ; 93,6 \times 58,5 \mu$.

Chez certains exemplaires, les lobes antérieurs de l'organe tribocytique sont saillants ; chez d'autres, non. Chez certains exemplaires, les vitellogènes masquent complètement la ventouse orale, le pharynx, la ventouse ventrale ; chez d'autres, ils laissent voir en partie, mais difficilement, ces organes. Chez certains exemplaires, les vitellogènes pénètrent dans la paroi de la poche copulatrice ; chez d'autres, non. 


\section{Discussion :}

Les caractères tirés de l'extension antérieure et postérieure des vitellogènes sont considérés par G. Dubois $(1938$, p. 68, 89) comme permettant de distinguer $S$. strigis (Schrank) de $S$. falconis Viborg-Szidat, mais il y a des variations individuelles qui interviennent, et, si l'on n'examine pas de nombreux exemplaires, il arrive que la moindre extension des vitellogènes de falconis ne puisse pas être nettement constatée.

Les œufs sont, en moyenne, plus petits chez falconis que chez strigis. Pour strigis, Dubois (1938, p. 89) indique 100-130 × 65-90, et, d'après Szidat, $120-130 \times 70-75 \mu$. Pour falconis, Dubois (1938, p. 69) indique 75-108 $\times$ 45-72, et, d'après Szidat, 80-90 $\times$ 50-55 $\mu$. Les dimensions que nous avons mesurées concordent avec celles de falconis, et le fait que l'hôte est un Accipitriforme confirme qu'il s'agit de falconis.

\section{Résumé et Conclusions}

Parmi les 11 espèces de Trématodes digénétiques récoltées en Iran par Y. Golvan et J. Rioux, il n'y en a pas de nouvelle pour la science ; néanmoins, elles montrent que la faune trématodologique iranienne est nettement et seulement paléarctique, sans apport de la faune indienne.

\section{Bibliographie}

AbildgaARd (Peter-Christian) (1806). - Zoologia Danica seu animalium Daniae et Norvegiae rariorum ac minus notorum descriptiones et historia. Volumen quartum, auctore Othone Friderico Müller, descripserunt et tabulae dederunt, P.C. Abildgaard... Havniae 1806, 46 p., pl. CXXI-CLX, f ${ }^{\circ}$.

BAer (Jean-Georges) (1924). - Description of a new genus of Lepodermatidae (Trematoda) with a systematic essay on the family. Parasitology, XVI, $\mathrm{n}^{\circ} 1,31$ janvier 1924, p. 22-31, fig. 1-2.

Baylis (Harry-Arnold) et Cannon (H. Graham) (1924). - A new Trematode from the Grass-snake. Ann. and Mag. Nat. History, ser. 9, vol. XIII, febr. 1924, p, 194-196, pl. VI.

- - (1924), - Further note on a new Trematode from the Grass-snake. Ann. and Mag. Nat. History, ser. 9, vol. XIII, may 1924, p. 558-559.

BHALÉRAo (G. D.) (1926). - On the Trematode parasites of a water-snake, Tropidonotus piscator. Parasitology, vol. XVIII, $\mathrm{n}^{\circ}$ 1, janvier 1926, p. 4-13, fig. 1-2.

- (1936). - Studies on the Helminths of India, Trematoda II. Journ. of Helminthology, vol. XIV, $\mathrm{n}^{\circ} 6$, décembre 1936, p. 181-206, fig. 1-5.

Bychovskaya-Pavlovskaya (Irina) (1962). - Trématodes des Oiseaux de la faune de l'U.R.S.S. Ouvrage écologo-géographique. Edition de l'Académie des Sciences de l'U.R.S.S. Institut zoologique, Laboratoire d'Helminthologie, Moscou-Léningrad 1962, 407 p., 107 fig.

Chisng (C. P.) (1951). - A comparative study of two species of Encyclometra metacercariae and their development in experimental hosts (Trematoda Plagiorchiidae). Lingnan Science Journal, vol. XXIII, $\mathrm{n}^{\circ}$ 3, 1951, p. 201-215, pl. XVIII-XXI, fig. 1-23.

DAwEs (Ben) (1946). - The Trematoda with special reference to british and other european form. XIV +644 p., fig. $1 \mathrm{~A}-81 \mathrm{H}$, Cambridge University Press, $1946,8^{\circ}$. 
Diesing (Carolus Mauritius) (1850). - Systema Helminthum. Vol. 1, Vindobonae 1850, XIII + $2+$ 679 p. + corrigenda 1 p.

Dollfus (Robert-Ph.) (1924). - Qu'est-ce que Distoma subflavum Sonsino ? Bull. Soc. Pathol. Exotique, Paris, t. XVII, $\mathrm{n}^{\circ}$ 7, séance du 9-7-1924, p. 572-577, 1 fig.

- Sur un distome de Tropidonotus natrix (L.) (1924). - Bull. Soc. zool. France, XLIX, n ${ }^{\circ} 3$, 20-7-1924, p. 268-276, fig. 1-8.

- (1929). - Sur le genre Telorchis. Ann. Parasitol. hum. et comp., t. VII, , $\mathrm{n}^{\circ}$ 1, 1-1-1929,

- $\quad$ p. 29-54, fig. 1-7 et t. VII, $\mathrm{n}^{\circ} 2,1-3-1929$, p. 116-132.

- (1948). - Sur deux Monostomes (Cyclocoelidae) pourvus d'une ventouse ventrale; observations sur la classification des Cyclocoeloidea, Albert Henry 1923; liste de leurs hôtes, répartition géographique. Ann. Parasitol. hum. et comp., t. XXIII, n 3-4, 31-12-1948, p. 129-199, fig. 1-14.

- (1958). - Miscellanea helminthologica maroccana XXIX. Deux espèces de distomes gen. Pleurogenoïdes et Opisthioglyphe, de l'intestion de Bufo du Moyen Atlas. Arch. Inst. Pasteur du Maroc, Casablanca, t. V, cahier VIII, 31-5-1958, p. 563-574, fig. 1-6

- (1958). - Opisthioglyphe endoloba (F. Dujardin 1845) est une espèce distincte d'Opisthioglyphe ranae (Froelich 1791). Ann. Parasitol. hum. et comp., t. XXXIII, n 3, 16-9-1958, p. 218-226, fig. 1-6.

- (1960). - Groupement des espèces dans la sous-famille Opisthioglyphinae R.-Ph. Dollfus, 1949. Libro Homenaje al $D^{r}$ Eduardo Caballero y C., ,Mexico 1960 p. 113-117.

Dubinina (M.-N.) (1945). - Etude écologique de la parasitofaune de Rana ridibunda du delta de la Volga. Analyse des travaux effectués dans la Section des Sciences biologiques de l'Académie des Sciences de 1941 à 1943, Moscou-Leningrad 1945, p. 168-169.

- (1950). - Etude écologique de la parasitofaune de la grenouille lacustre (Rana ridibunda Pall.) du delta de la Volga. Parasitologitch Sbornik Zoologitch. Instituta Akadem. Nauk C.C.C.P., t. XII, 1950, p. 300-350, fig. 1-12 b.

Dubors (Georges) (1938). - Monographie des Strigeida (Trematoda). Mém. Soc. Neuchâteloise des Sciences naturelles, t. VI, 25-5-1938, p. 1-535, fig. 1-540 + fig. A-N.

- (1959). - Révision des Cyclocoelidae Kossack 1911. Revue Suisse de Zoologie, t. LXVI, fasc. $1, \mathrm{n}^{\circ} 2$, avril 1959 , p. $67-147$, fig. $1-11$, pl. I-V.

DUjardin (Félix) (1845). - Histoire Naturelle des Helminthes ou Vers intestinaux. Nouvelles suites à Buffon, $42^{\circ}$ livraison, Paris, Roret, 1845 , XVI +654 p.; Atlas 15 p., pl. I-XII.

EdelÉNYI (Béla) (1962). - Zur Kenntniss der Endoparasiten unserer Reptilien. Az Egri Pedagogiai Foiskôla Fuzetei, n ${ }^{\circ}$ 272, Eger 1962, p. 561-578, fig. 1-13. En hongrois, résumé en allemand.

ErColani (Giambattista) (1881). - Dell'adattamento della specie all'ambiente. Nuove ricerche sulla storia genetica dei Trematodi. Memorie dell' Accademia delle scienze dell'Instituto di Bologna, ser 4, t. II, 10-2-1881, p. 239-334, pl. I-III.

Fedorova (O.-E.) (1954). - La variabilité des caractères morphologiques et leur importance dans la systématique du genre de Trématodes Plagiorchis Lühe, 1899. Résumé d'auteur d'une Dissertation présentée pour la canditature en Sciences biologiques, Léningrad 1954, 13 p.

Gupta (N.-K.) (1954). - On Encyclometra vitellata, a new species from water-snake Natrix piscator. Research Bulletin Panjab University. Zoology, $\mathrm{n}^{\circ}$ 57, sept. 1954, p. 139-241, fig. 1.

GuYÉNOT (Emile) et NAville (André) (1924). - Glugea encyclometrae n. sp. et G. ghigii n. sp. parasites de Platodes et leur développement dans l'hôte vertébré (Tropidonotus natrix L.). Revue Suisse de Zoologie, t. XXXI, $\mathrm{n}^{\circ}$ 3, mai 1924, p. 75-115, fig. 1-11, pl. II, fig. 1-11 pl. III, fig. 12-17.

HOUDEMER (Fernand-Eugène) (1927). - Observations et recherches sur l'hygiène et la pathologie animales au Tonkin. Paris, 1927, 8, 312 p. 
Hugues (R.-Chester), Higginbotham (Joe-W.) et Clary (Jasper-W). (1941-1942). - The Trematodes of Reptiles. Part. II. Host Catalogue. Proceed. Oklahoma Acad. of Science, v. XXI (1940) 1941, p. 35-43 ; - Part I. Systematic Section. American Midland Naturalist, Notre-Dame, Indiana, v. XXVII, $\mathrm{n}^{\circ}$ 1, jan. 1942, p. 109-134 ; - Part III. Conclusion. Proceed. Oklahoma Acad. of Science, v. XXII (1941) 1942, p. 90-114.

Joyeux (Charles) et Houdemer (Fernand-Eugène) (1928). - Recherches sur la faune helminthologique de l'Indochine (Cestodes et Trématodes). Ann. Parasitol. hum. et comp., t. V, n 4, 1-10-1927 et t. VI, $\mathrm{n}^{\circ} 1,1-1-1928$, p. 289-309, fig. 1-6, p. 27-58, fig. 7-14.

Joyeux (Charles) et Gaud (Jean) (1945). - Recherches helminthologiques marocaines. Arch. Inst. Pasteur Maroc, Casablanca, t. III, cahier 4, 1945, p. 111-143, fig. 1-6 c.

Leonov (V.-A.) (1958). - Nouveaux Trématodes d'Oiseaux. Travaux sur l'helminthologie pour les 80 ans de l'Académicien K.-I. Skrjabin. Moscou 1958. Edition de l'Académie des Sciences de l'U.R.S.S., p. 200-203, fig. 1-3.

Looss (Arthur) (1894). - Distomen unsere Fische und Frosche. Bibliotheca Zoologica Leipzig, Bd VI (1894), Heft $16,4^{\circ}$, p. 1-296, fig. texte, pl. I-IX, fig. 1-192.

- (1899). - Weitere Beiträge zur Kenntniss der Trematodenfauna Aegyptens. Zoolog. Jahrbücher System., Bd XII, Heft 5-6, 28-12-1899, p. 521-784, fig. texte A-B, pl. XXIV-XXXII, fig. 1-90.

LüHE (Max) (1906). - Ueber Ostpreussens Helminthenfauna. Schriften der Physik. ôkonom. Gesellschaft zu Königsberg in Preussen, Jahrg. XLVII, 1906, p. 133-137.

- (1909). - Trematodes. Süsswasserfauna Deutschlands, Heft 17, Jena 1909, 217 p., 188 fig.

Massino (B.-G.) (1924). - Sur la connaissance des Nématodes d'Emys orbicularis de l'Araxe. Laboratoire de parasitologie de l'Institut de médecine vétérinaire de Moscou, 13-9-1924, p. 1-12, fig. 1-5.

- (1927) Die Bestimmung der Arten der Gattung Plagiorchis Lühe. Sammlung helminthologischer Arbeiten Prof. D ${ }^{r}$ K-I Skrjabin von seinen Schülern gewidmet. Moscou 1927, p. 108-113, fig. 1-6.

Merra (H.-R.) (1931). - Two distomate Trematodes from Indian Reptiles. The Allahabad University Studies, v. VIII, Science Section, 1931, p. 31-52, fig. texte 1-2, pl. I-VI, fig. 1-6.

MENDHEIM (Hans) (1943). - Beitrâge zur Systematik und Biologie der Familie Echinostomatidae. Archiv für Naturgeschichte, N.-F., Bd XII, Heft 2, 1943, p. 175-302.

Mödlinger (Gustáv) (1924). - Neue Trematoden der ungarischen Fauna. Mat. és Term. tud. Ertesitö, XLI Kötet. III tablá. 1924 A.M.T. Akad. III osztálya 1924, majus hó 19-én tartott üléséböl, p. 193-197+1 p. (en allemand), pl. fig. 1-5.

MüHLING (Paul) (1898). - Die Helminthen-Fauna der Wirbeltiere Ostpreussens. Arch für Naturgeschichte, LXIV Jahrg., Bd I, Heft 1, Mai 1898, p. 1-118, pl. I-IV, fig. 1-28.

Odening (Klaus) (1959). - Ueber Plagiorchis, Omphalometra und Allocreadium. Zeitschr. f. Parasitenkunde, Bd XIX, Heft 1, 17-3-1959, ,p. 14-34, fig. 1-7.

- 1960 Der Ansatzmodus des Excretionsgefässystems und die systematische Stellung von Encyclometra (Trematoda Digenea). Monatsberichte der deutschen Akad. der Wissensch. zu Berlin, Bd. II, Heft 7, 1960, p. 445-449, fig. 1-2.

- (1960). - Das Exkretionssystem von Telorchis assula (Dujardin 1845) (Trematoda, Ochetosomatoidea). Zeitschr. f. Parasitenkunde, Bd XX, Heft 4, 25-11-1960, p. 335-344, fig. 1-2.

- (1961). - Zur morphologischen. Variationbreite von Plagiorchis und Opisthioglyphe (Trematoda, Digenea). Monatsber. d. deutschen Akad. Wissensch. zu Berlin, Bd III, Heft $3 / 4,1961$, p. $188-201$, fig. $1-27$.

ODHNER (Theodor) (1902). - Trematoden aus Reptilien, nebst allgemein systematischen Bemerkungen. Oefversigt af Kungl. Svenska Vetenskaps-Akademiens Förhandlingar, Stockholm, Arg LIX, $\mathrm{n}^{\circ} 1,8-1-1902$, p. 19-45, fig. 1-3. 
OLsson (Peter-J.) (1876), - Bidrag till Skandinaviens Helminthfauna I. Kongl. Svenska Vetensk. Akad. Handlingar, Stockholm, Bd XIV, $\mathrm{n}^{\circ} 1,1876$, p. 1-35, pl. I-IV, fig. 1-71.

PARK (James-T.) (1940). - Trematode parasites of Reptilia from Tyôsen. I Three new digenetic Trematodes, Encyclometra koreana sp. nov., Neomicroderma elongata gen. nov. sp. nov., (Plagiorchidae) and Proalarioides kobayashii sp. nov. (Strigeidae). Keijo Journ. of Medicine, vol. $\mathrm{X}, \mathrm{n}^{\circ} 3, \ldots 1940$ p. 113-123, texte 1-3, pl. XI, fig. 1-9.

PARONA (Corrado) (1896). - Intorno ad alcuni Distomi nuovi o poco noti. Bollett. dei Musei di Zool. e Anat. comp. della $R$. Univ. di Genova, 1896, $\mathrm{n}^{\circ}$ 50, p. 1-19, fig. 1-7.

Poirier (Justin) (1886). - Trématodes nouveaux ou peu connus. Bull. Soc. Philomatique de Paris, $7^{\circ}$ s., t. X, 1886 (séance du 28-11-1885), p. 20-40, pl. I-IV.

Polonio (Antonio-Frederico) (1859). - Prospectus helminthum qui in reptilibus et amphibiis faunae italicae continentur. Padova 1859, $8^{e}, 20 \mathrm{p}$.

RUdolPHI (Carl-Asmund) (1819). - Entozoorum synopis cui accedunt mantissa duplex et indices locupletissimi. Berolini 1819, $\mathrm{x}+811$ p., pl. I-III.

RUszkowski (Jerzy-Stanislaw) (1926). - Telorchis gabesensis n. sp. parasite de la tortue africaine Clemmys leprosa Schweigg. Ann. Parasitol. hum. et comp., t. IV, $\mathrm{n}^{\circ} 4$, oct. 1926, p. 327-329, fig. 1.

SKRJABIN (Constatin-Ivanovitch) (1924). - Compte rendu de l'étude helminthologique de l'Arménie. Travaux de l'Institut tropical d'Arménie, 1924, $\mathrm{n}^{\circ} 1$, p. 9-11.

- (1924). - Trématodes d'Emys orbicularis, vallée du fleuve Araxe. Travaux de l'Institut tropical d'Arménie, $1924, \mathrm{n}^{\circ} 1$, p. 34-40, fig. 1-2.

- $\quad$ et Popov (N.-P.) (1924), - Compte rendu des travaux helminthologiques de l'expédition en Arménie pendant l'année 1923. Journal russe de Médecine tropicale, t. I, n 2, 1924, p. 58-63.

- (1925). - Sur les Trématodes d'Emys orbicularis L. Ann. Parasitol. hum. et comp., t. III, $\mathrm{n}^{\circ} 3$, juillet 1925 , p. 281-289, fig. 1-2.

- et Povov (N.-P.) (1927). - The 10th Helminthological Expedition to Armenia, 1923, 10/VII-3/XII/1923. The Results of Research of twenty-eight Helminthological Expeditions in U.R.S.S. (1919-1925) under the direction of Prof. K.I. Skrjabin. Moscou 1927, p. 133143, 1 pl. Résumé en anglais, p. 280.

- et Baschrirova (E. Ya.) (1956). - Famille Echinostomatidae Dietz 1909. Trématodes de l'Homme et des Animaux, t. XII, p. 51-932, fig. 13-312. Edition de l'Acad. des Sciences de l'U.R.S.S., Moscou 1956.

Sonsino (Prospero) (1892). - Dei Distomi dello Zamenis viridiflavus, Lacép. et di una fase del ciclo vitale di uno di essi. Atti della Soc. Toscana di Scienze Naturali, Processi verbali, Pisa, vol. VII, adunanza 15.5 .1892 , p. 91-95.

Stossich (Michele) (1895). - I. Distomi dei Rettili. Bollettino della Società Adriatica di Scienze Naturali in Trieste, vol. XVI, 1895, p. 213-239.

- (1904). - Alcuni Distomi della collezione elmintologica del Museo zoologico di Napoli. Annuario del Museo Zoologico della Univ. di Napoli, nuova serie vol. I, $\mathrm{n}^{\circ}$ 23, 23.6.1904, p. 1-14, pl. II, fig. 1-3.

Trmofeev (N. E.) (1900). - Les Trématodes des amphibiens et reptiles des environs de Kharkow. Travaux de la Société des Naturalistes à l'Université impériale de Kharkow, t. XXXIV, 1900 , p. $137-166$, pl. I, fig. 1-4.

Verma (S. C.) (1935), - Studies on the indian species of the genus Echinochasmus, Part I. And on an allied new genus Episthochasmus. Proceed. Indian Acad. of Sciences, vol. I, $\mathrm{n}^{\circ} 12$, june 1935 , p. 837-856, fig. 1-11.

VoJTKova (Ludmila) (1961). - Beitrag zur Kenntnis der Helminthenfauna der Frösche in der Umgebung von Komarno. Biológia, Casopis slovenskej .4kad. Vied, t. XVI, $\mathrm{n}^{\circ} 1$, Bratislava 1961, p. 25-30, fig. 1-9. 
WaLlace (Franklin, Gerhard) (1936). - Two new Trematode Parasites of Canton snakes (Trematoda: Lepodermatidae). Lingnan Science Journal, v. XV, $\mathrm{n}^{\circ} 3,24.7 .1936$, p. 355-364, fig. 1-7.

Yamagurti (Satyû) (1933). - Studies on the Helminth Fauna of Japan. Part 1. Trematodes of Birds, Reptiles and Mammals. Japanese Journ. of Zoology, t. V, $\mathrm{n}^{\circ}$ 1, 15-8-1933, p. 1-134, fig. 1-57.

YEH (Liang-Shen) (1958). - A review of the Trematode genus Encyclometra Baylis and Cannon, 1924. Journ. of Helminthology, vol. XXXII, $\mathrm{n}^{\circ} 1 / 2$, 1958, p. 99-114, fig. 1-9.

Yoshida (Sadao) et OzAKI (Yoshimasa) (1929). - A new Trematode, Encyclometra japonica, nov. sp. from the snake, Elaphe quadrivirgata. Annotationes zoologicae japonenses, vol. XII, $\mathrm{n}^{\circ} 1,25.7 .1929$, p. 239-243, pl. I, fig. 1-3.

(Muséum National d'Histoire Naturelle, 57, rue Cuvier, Paris, 5*) 